\title{
TRPC6 fulfills a calcineurin signaling circuit during pathologic cardiac remodeling
}

\author{
Koichiro Kuwahara, ${ }^{1}$ Yanggan Wang, ${ }^{2}$ John McAnally, ${ }^{1}$ James A. Richardson, ${ }^{1,3}$ \\ Rhonda Bassel-Duby, ${ }^{1}$ Joseph A. Hill, ${ }^{1,2}$ and Eric N. Olson'
}

\author{
1Department of Molecular Biology, ${ }^{2}$ Department of Internal Medicine, and 3Department of Pathology, \\ University of Texas Southwestern Medical Center at Dallas, Dallas, Texas, USA.
}

\begin{abstract}
The heart responds to injury and chronic pressure overload by pathologic growth and remodeling, which frequently result in heart failure and sudden death. Calcium-dependent signaling pathways promote cardiac growth and associated changes in gene expression in response to stress. The calcium/calmodulin-dependent phosphatase calcineurin, which signals to nuclear factor of activated T cells (NFAT) transcription factors, serves as a transducer of calcium signals and is sufficient and necessary for pathologic cardiac hypertrophy and remodeling. Transient receptor potential (TRP) proteins regulate cation entry into cells in response to a variety of signals, and in skeletal muscle, expression of TRP cation channel, subfamily C, member 3 (TRPC3) is increased in response to neurostimulation and calcineurin signaling. Here we show that TRPC6 was upregulated in mouse hearts in response to activated calcineurin and pressure overload, as well as in failing human hearts. Two conserved NFAT consensus sites in the promoter of the TRPC6 gene conferred responsiveness to cardiac stress. Cardiac-specific overexpression of TRPC6 in transgenic mice resulted in heightened sensitivity to stress, a propensity for lethal cardiac growth and heart failure, and an increase in NFAT-dependent expression of $\beta$-myosin heavy chain, a sensitive marker for pathologic hypertrophy. These findings implicate TRPC6 as a positive regulator of calcineurin-NFAT signaling and a key component of a calcium-dependent regulatory loop that drives pathologic cardiac remodeling.
\end{abstract}

\section{Introduction}

The adult heart responds to diverse stress signals - such as hypertension, myocardial infarction, pressure overload, and abnormalities in contractility and structure due to inherited gene mutations - by hypertrophic growth and remodeling, which often progress to heart failure and sudden death (reviewed in refs. 1-3). These pathologic changes are accompanied by a reversion of the adult cardiac gene program to a more "fetal" state. A hallmark of such transcriptional remodeling is the downregulation of $\alpha$-myosin heavy chain ( $\alpha$-MHC), which possesses high ATPase activity, and the concomitant upregulation of $\beta$-MHC, which possesses relatively low ATPase activity, and consequent diminution of cardiac contractility (4). There has been great interest in deciphering the intracellular signaling pathways that control pathologic cardiac growth and, more specifically, that govern the $\alpha$-MHC to $\beta$-MHC switch because of the potential therapeutic benefit of manipulating these mechanisms.

Abnormalities in intracellular $\mathrm{Ca}^{2+}$ signaling initiate and sustain pathologic cardiac hypertrophy and fetal gene activation (5-7), but many details of the mechanisms involved in these processes remain to be elucidated. Extracellular stimuli modulate intracellular $\mathrm{Ca}^{2+}$ levels by promoting $\mathrm{Ca}^{2+}$ release from intracellular organelles or

Nonstandard abbreviations used: ANP, atrial natriuretic peptide; BNP, brain natriuretic peptide; $\mathrm{BW}$, body weight; $\mathrm{CnA} \triangle \mathrm{C}$, constitutively active calcineurin; ET-1, endothelin-1; HW, heart weight; MHC, myosin heavy chain; -3963-MHC-luc, -396 bp promoter region of the mouse $\beta$-MHC gene fused to a luciferase reporter; NFAT, nuclear factor of activated T cells; NFATc $4 \Delta 317$, constitutively active NFATc4; PE, phenylephrine; RCAN1, regulator of calcineurin 1; SERCA2, sarco/endoplasmic reticulum $\mathrm{Ca}^{2+}$-ATPase isoform 2; SOCE, store-operated $\mathrm{Ca}^{2+}$ entry; $\mathrm{TAB}$, thoracic aortic banding; TRP, transient receptor potential; TRPC, TRP cation channel, subfamily C. Conflict of interest: E.N. Olson is a scientific founder and consultant of Myogen Inc. Citation for this article: J. Clin. Invest. 116:3114-3126 (2006). doi:10.1172/JCI27702. entry of $\mathrm{Ca}^{2+}$ across the plasma membrane via agonist- or receptor-activated $\mathrm{Ca}^{2+}$ entry channels, voltage-gated $\mathrm{Ca}^{2+}$ channels, or ligand-gated cation channels. Receptor-activated $\mathrm{Ca}^{2+}$ entry, including store-operated $\mathrm{Ca}^{2+}$ entry (SOCE), is initiated upon binding of ligands to $\mathrm{G}$ protein-coupled receptors or receptor tyrosine kinases, leading to activation of phospholipase C (PLC) and phosphatidylinositol turnover $(8,9)$. SOCE participates in the signaling pathways leading to cardiac hypertrophy $(10,11)$.

The serine-threonine phosphatase calcineurin functions as a $\mathrm{Ca}^{2+}$-dependent regulator of cardiac hypertrophy and the fetal gene program (12). Calcineurin dephosphorylates and induces nuclear translocation of the nuclear factor of activated $\mathrm{T}$ cells (NFAT) family of transcription factors, which bind the regulatory regions of cardiac genes in conjunction with other transcription factors and promote hypertrophic growth (13). Cardiac expression of constitutively active forms of calcineurin A or NFATc4 in Tg mice is sufficient to elicit a pathologic cardiac growth response (12). Conversely, pharmacologic or genetic blockade of calcineurin-NFAT signaling suppresses cardiac growth in response to diverse pathologic stimuli (12, 14-19).

In $\mathrm{T}$ cells, $\mathrm{Ca}^{2+}$ release-activated $\mathrm{Ca}^{2+}$ (CRAC) channels, the archetypal version of SOCE, play a crucial role in the sustained activation of NFAT $(20,21)$. Transient receptor potential (TRP) proteins are candidate channel subunits responsible for receptoractivated $\mathrm{Ca}^{2+}$ entry and $\operatorname{SOCE}(8,22)$. Among the 7 subfamilies of mammalian TRP channels $(8,9)$, the TRP cation channel, subfamily C (TRPC) members are further divided into 4 subgroups based on their primary amino acid sequences: TRPC1; TRPC2; TRPC4 and -5 ; and TRPC $3,-6$, and -7 (8). TRPC $1,-4$, and -5 or TRPC $3,-6$, and -7 physically interact and form heteromultimeric complexes $(9,23,24)$. TRPC $3,-6$, and -7 , which are $75 \%$ identical, couple receptor-PLC signaling pathways to $\mathrm{Ca}^{2+}$ entry $(8,25)$. Recently, 
A
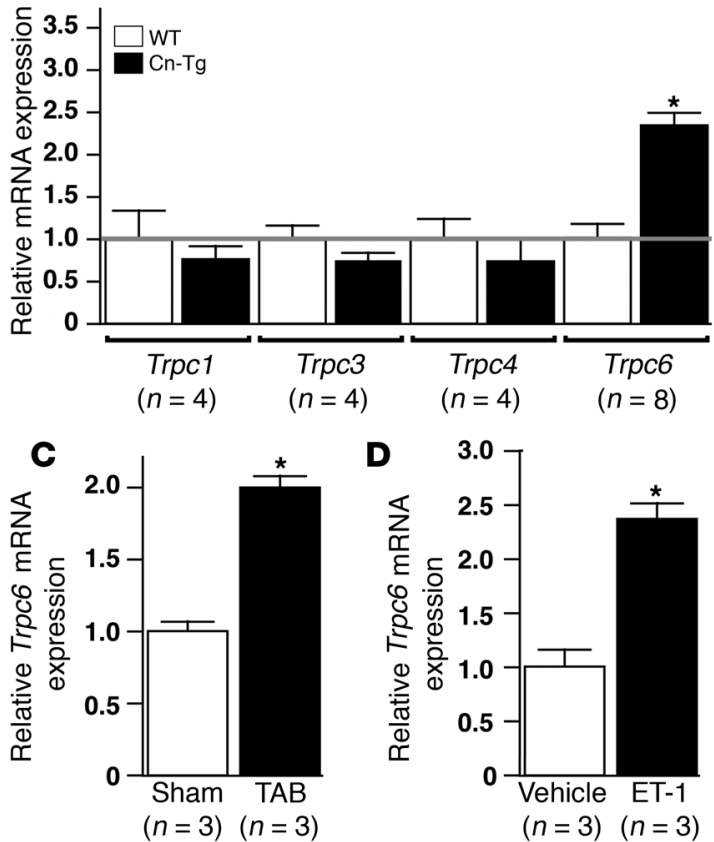

B
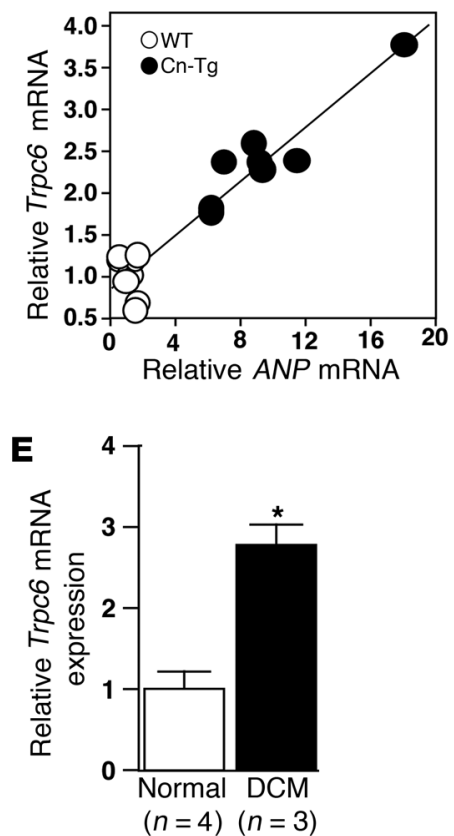

Figure 1

Increased mRNA expression of TRPC6 in models of cardiac hypertrophy and human failing heart. (A) RNA was isolated from hearts of WT and calcineurin $\mathrm{Tg}$ mice $(\mathrm{Cn}-\mathrm{Tg})$ at 10 weeks of age, and mRNA levels of Trpc1, $-3,-4$, and -6 determined by real-time RT-PCR as described in Methods. Shown are mRNA levels relative to WT normalized by $18 \mathrm{~S}$ RNA levels. ${ }^{*} P<0.05$ versus WT. (B) The correlation between Trpc6 and ANP mRNA expression in ventricles was examined by linear regression analysis. $r=0.953$. $P<0.0001$. (C) Trpc6 mRNA levels were determined by real-time RT-PCR in RNA isolated from hearts of mice subjected to TAB or sham operation for 3 weeks. Shown are mRNA levels relative to sham-operated ventricles normalized by $18 \mathrm{~S}$ RNA levels. ${ }^{*} P<0.05$ versus sham. (D) Trpc6 mRNA levels were determined by real-time RTPCR in RNA from primary neonatal rat ventricular myocytes treated with or without ET-1 for 24 hours. Shown are mRNA levels relative to vehicle-treated controls normalized by $18 \mathrm{~S}$ RNA levels. ${ }^{*} P<0.05$ versus vehicle. (E) TRPC6 mRNA levels were determined by real-time RT-PCR in total RNA isolated from human hearts with dilated cardiomyopathy (DCM) or normal human hearts. Shown are mRNA levels relative to normal hearts (assigned as 1.0) normalized by $18 \mathrm{~S}$ levels. ${ }^{\star} P<0.05$ versus normal hearts.

TRPC3 channels were reported to be involved in NFAT activation in skeletal muscle cells (26).

In this study, we examined the potential involvement of TRPC channels in cardiac calcineurin-NFAT signaling. We found that Trpc6 expression was upregulated in the hearts of mice expressing constitutively active calcineurin and subjected to pressure overload by thoracic aortic banding (TAB). Consistent with these findings, the promoter of the Trpc6 gene contains 2 conserved NFAT-binding sites that confer responsiveness to calcineurin-NFAT signaling. Cardiac-specific overexpression of TRPC6 in Tg mice sensitized the heart to stress when expressed at low levels and resulted in fatal cardiomyopathy when expressed at high levels. TRPC6 expression was coupled to a pronounced increase in expression of $\beta$-MHC, a sensitive marker for pathologic hypertrophy. Similarly, overexpression of TRPC6 in cardiomyocytes activated the NFAT-dependent promoter of the regulator of calcineurin 1 (RCAN1) gene (previously called modulatory calcineurin-interacting protein 1 [MCIP1]), and siRNA knockdown of TRPC6 reduced hypertrophic signaling by phenylephrine (PE) and endothelin-1 (ET-1), suggesting that TRPC6 is involved in activation of the calcineurin-NFAT pathway in response to $G$ protein-coupled receptor signaling. These findings demonstrate that TRPC6 provides a positive regulatory circuit for calcineurin-NFAT signaling during pathologic cardiac remodeling and activation of $\beta$-MHC gene expression.

\section{Results}

Increased expression of TRPC6 in hypertrophic and failing hearts. To investigate whether changes in TRPC expression participate in calcineurin-dependent cardiac growth (12), we examined the expression of Trpc transcripts by realtime RT-PCR in hearts from $\mathrm{Tg}$ mice harboring an $\alpha$-MHC-calcineurin transgene, which results in profound cardiac hypertrophy. We detected cardiac expression of $\operatorname{Trpc1},-3,-4$, and -6 , and among these, Trpc6 mRNA was significantly upregulated in ventricles of calcineurin Tg mice (Figure 1A). As TRPC2 is a pseudogene in humans (8), we did not test its expression. Among other TRPC family members, $\operatorname{Trpc5}$ and -7 transcripts were not detected in the heart, consistent with previous studies $(27,28)$.

Expression of Trpc6 mRNA correlated closely with that of atrial natriuretic peptide (ANP), a sensitive marker of cardiac stress and hypertrophy (Figure 1B). Cardiac expression of Trpc6 was also upregulated in mice subjected to TAB (Figure 1C). In addition, Trpc6 mRNA was increased in cultured rat ventricular myocytes in response to ET-1, a potent prohypertrophic agonist (Figure 1D). TRPC6 mRNA expression was also augmented in hearts of human patients with dilated cardiomyopathy compared with nonfailing hearts (Figure 1E). We conclude that TRPC6 is upregulated in response to diverse pathologic stimuli that promote cardiac hypertrophy and remodeling.

TRPC6 is directly regulated by calcineurin-NFAT signaling. To begin to define the mechanism that regulates TRPC6 expression during pathologic cardiac growth, we analyzed the 5 ' flanking region of the mouse Trpc6 gene, which is highly conserved among different mammalian species. Within this region, we identified 2 sequences between -791 and -763 that resembled the NFAT consensus-binding site $([\mathrm{A} / \mathrm{T}] \mathrm{GGAAA}[\mathrm{A} / \mathrm{N}][\mathrm{A} / \mathrm{T} / \mathrm{C}] \mathrm{N})$, which were relatively conserved among humans and rodents (Figure $2 \mathrm{~A}$ ).

NFATc 4 translated in vitro bound the labeled NFAT-like sequences from the TRPC6 promoter, and binding was completely eliminated by the presence of unlabeled NFAT consensus sequences (Figure 2B). Similarly, unlabeled oligonucleotides representing either of the Trpc6 NFAT-like sequences effectively competed for binding of NFATc4 to a canonical NFAT consensus sequence (Figure 2B). The NFATc4-DNA complex was supershifted by an NFATc4 antibody, confirming the presence of NFATc4 in the complex. 
A

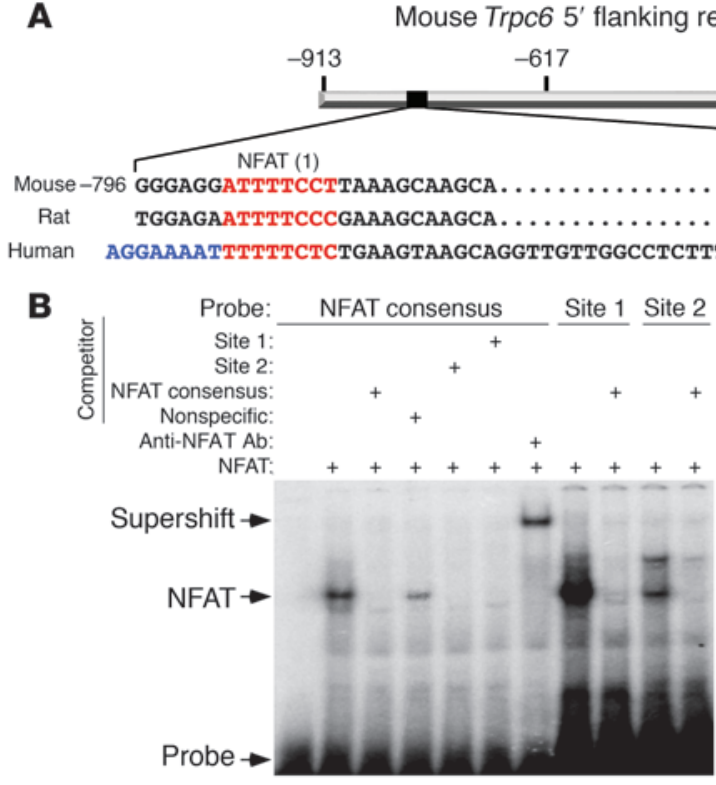

C
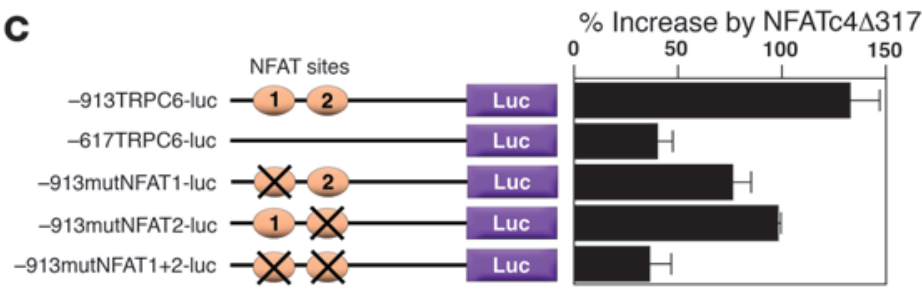

D

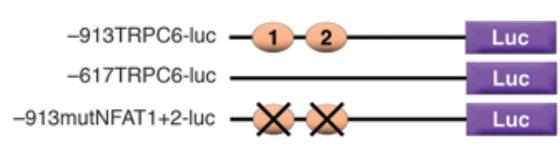

$\mathbf{E}$

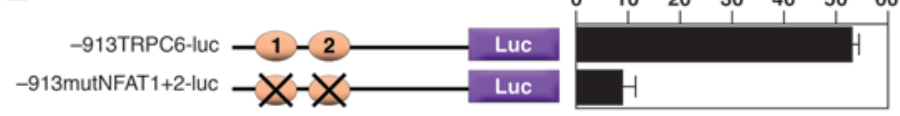

\section{Figure 2}

The calcineurin-NFAT pathway regulates Trpc6 gene transcription. (A) Schematic representation of the $5^{\prime}$ upstream region of the mouse Trpc6 gene. Sequences between -796 and $-750 \mathrm{bp}$ of the mouse Trpc6 gene are aligned with the corresponding sequences of rat Trpc6 and human TRPC6 genes. NFAT-like sites are shown in red and blue. (B) Gel mobility shift assay was performed using in vitro translated NFATc $4 \Delta 317$ and ${ }^{32 P}$-labeled probes of NFAT consensus sequences or NFAT-like sites 1 or 2 from the Trpc6 promoter. (C) Rat neonatal ventricular myocytes were cotransfected with an expression plasmid of NFATc $4 \Delta 317$ and -913TRPC6-luc, -617TRPC6-luc, or the Trpc6 promoter with NFAT site mutations (-913mutNFAT1-luc, -913mutNFAT2-luc, or -913mutNFAT1+2-luc). Percent increase in expression with NFATc4 4317 compared with empty pGL3 luciferase reporter vector is shown. (D) Rat neonatal ventricular myocytes were cotransfected with a plasmid expressing $\mathrm{CnA} \triangle \mathrm{C}$ and -913TRPC6-luc, -617TRPC6luc, or the Trpc6 promoter with 2 NFAT site mutations. Percent increase in expression with CnA $\triangle C$ compared with empty pGL3 luciferase reporter vector is shown. (E) Rat neonatal ventricular myocytes were cotransfected with either -913TRPC6 promoter region or luciferase reporter gene fused to the Trpc6 promoter with 2 NFAT site mutations in the presence or absence of $10 \mathrm{nM}$ ET-1. Percent increase in luciferase expression by ET-1 with each construct is shown.
To determine whether NFAT can activate the TRPC6 promoter, a DNA fragment extending to -913 bp relative to the translation initiation site of the mouse Trpc6 gene was fused to a luciferase reporter (-913TRPC6-luc) and cotransfected with an expression vector encoding constitutively active NFATc4 (NFATc4 $\Delta 317$ ) in cardiomyocytes. Transcriptional activity of the TRPC6 promoter was significantly increased in the presence of NFATc4, and deletion of the region containing the NFAT binding sites (deletion of -913 to -617 in the TRPC6 promoter; -617TRPC6-luc) reduced NFAT responsiveness. Site-directed mutations in either of the NFAT-like sites of the TRPC6 promoter partially reduced, and mutations of both sites nearly abolished, the response to NFATc $4 \Delta 317$ (Figure $2 \mathrm{C}$ ). Moreover, overexpression of constitutively active calcineurin $(\mathrm{CnA} \Delta \mathrm{C})$ activated the TRPC6 promoter, and deletion of the region comprising the NFAT sites or sitedirected mutations of both NFAT sites abolished the responsiveness of the promoter to calcineurin (Figure 2D). The TRPC6 promoter was also activated by ET-1, which activates calcineurin signaling, and the NFAT sites were required for this response (Figure 2E).
Increased expression of TRPC6 enhances NFAT activity. Because the TRPC family participates in receptor-mediated $\mathrm{Ca}^{2+}$ entry, which activates the calcineurin-NFAT pathway, we examined whether increased expression of TRPC6 in cardiomyocytes was capable of activating NFAT-dependent transcription. Indeed, overexpression of TRPC6 resulted in redistribution of NFATc4-GFP from the cytoplasm to the nucleus in transfected COS- 1 cells, as typically occurs in response to calcineurin activation (Figure 3, A and B). TRPC6 expression also activated a luciferase reporter controlled by the promoter region of exon 4 of the RCAN1 gene, which contains 15 NFAT sites (29), in transfected COS-1 cells (Figure 3C). RCAN1, a calcineurin inhibitor (16), inhibited activation of the RCAN1-luciferase reporter by TRPC6 (Figure 3C). TRPC6 overexpression also significantly increased RCAN1-luciferase activity in cardiomyocytes; ET-1 acted synergistically with TRPC6 to activate the reporter (Figure 3D), and RCAN1 blocked this activation (Figure 3E).

To examine the potential involvement of endogenous TRPC6 in hypertrophic signaling, cardiomyocytes were cotransfected with 

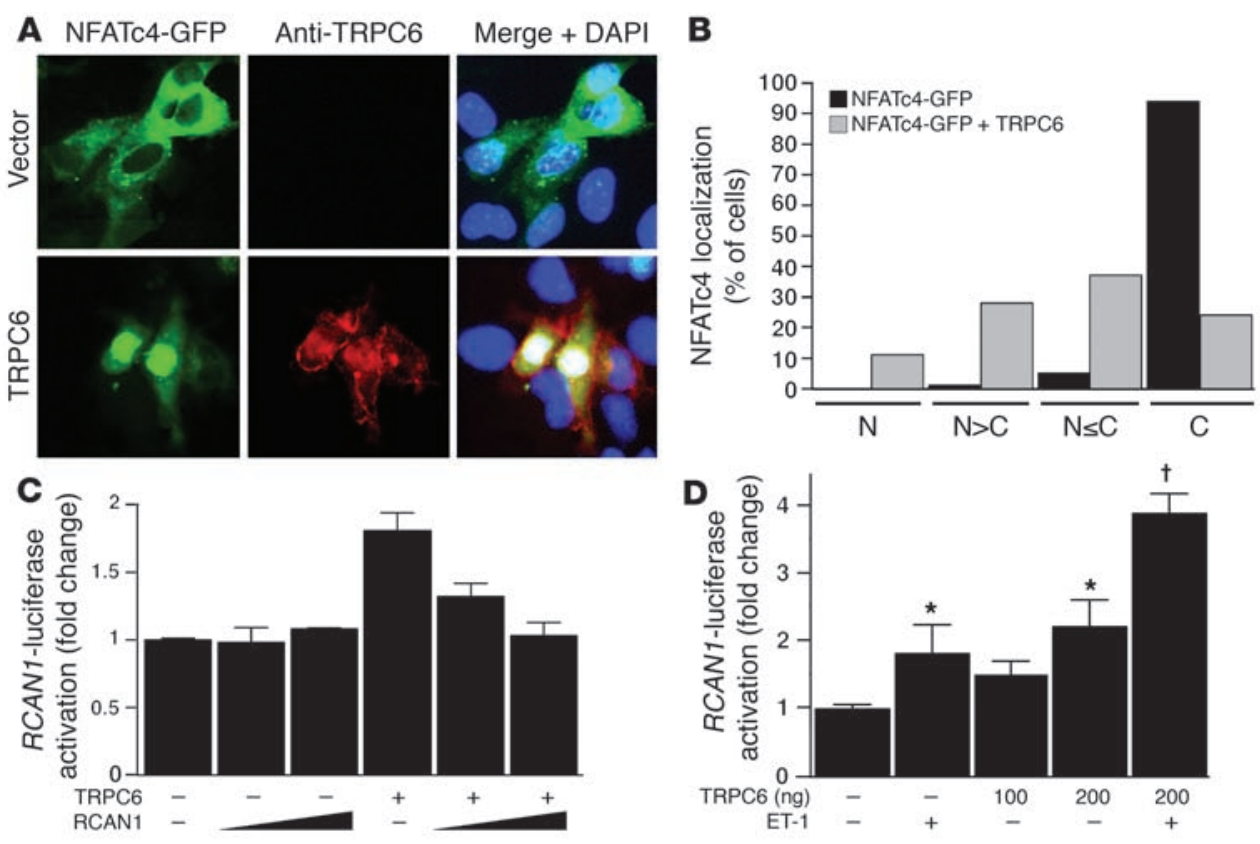

E
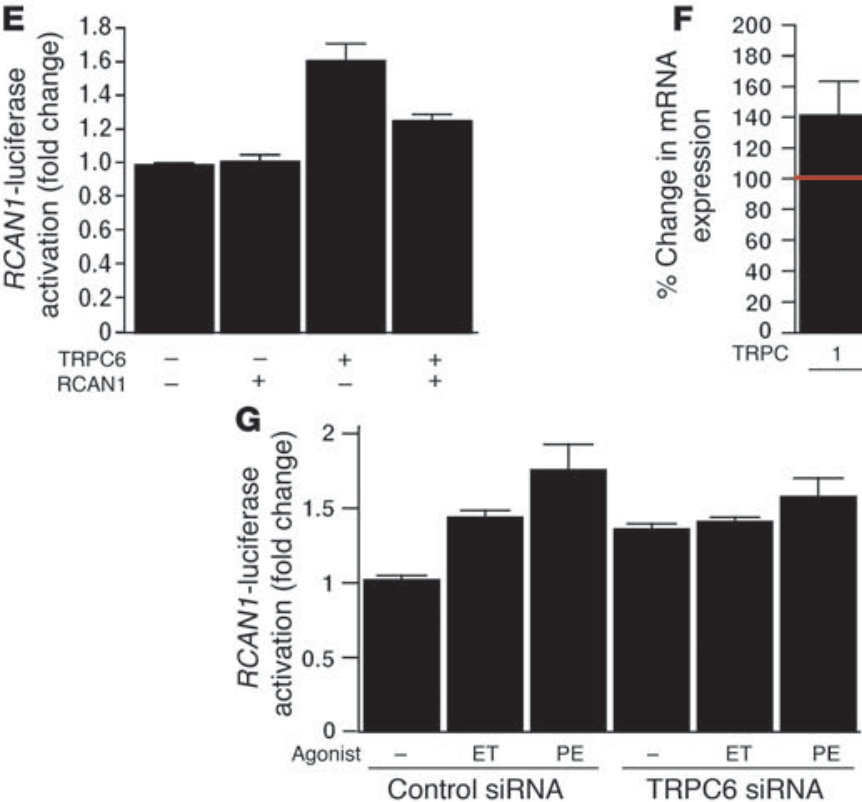

Figure 3
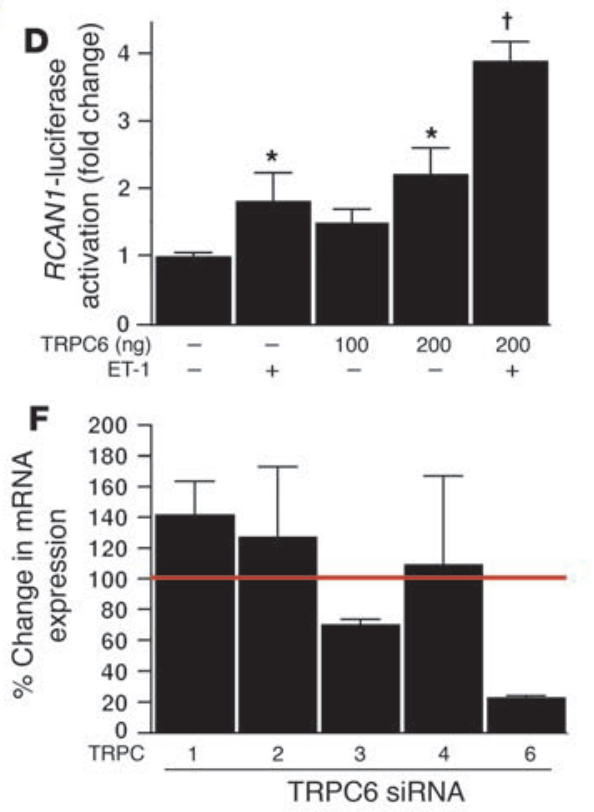

TRPC6 activates NFAT-dependent transcription in ventricular myocytes. (A) COS-1 cells were cotransfected with the expression vectors for NFATc4-GFP (green) and TRPC6 or empty vector, fixed, permeablized, and immunostained with anti-TRPC6 (red). Nuclei are stained blue (DAPI). Magnification, $\times 400$. (B) Effects of TRPC6 on NFATc4 subcellular distribution were quantified with microscopic examination of greater than 100 cells per condition. N, NFATc4-GFP localized exclusively in nucleus; N>C, nuclear NFATc4-GFP localization exceeds cytoplasmic; $\mathrm{N} \leq \mathrm{C}$, cytoplasmic NFATc4-GFP localization equals or exceeds nuclear; C, NFATc4-GFP localized exclusively in cytoplasm. (C) COS-1 cells were cotransfected with RCAN1-luciferase and TRPC6 and RCAN1 expression vectors. Fold activation over RCAN1-luciferase without expression plasmids is shown. (D) Myocytes were cotransfected with RCAN1-luciferase reporter plasmid and a plasmid expressing rat TRPC6 at various doses in the presence or absence of ET-1. Fold activation over RCAN1luciferase alone is shown. ${ }^{*} P<0.05$ versus control; ${ }^{\dagger} P<0.05$ versus control, ET-1, and TRPC6 (200 ng). (E) Myocytes were cotransfected with RCAN1-luciferase and plasmids expressing rat TRPC6 and RCAN1. Fold activation over $R C A N 1$-luciferase without expression plasmids is shown. ${ }^{*} P<0.05$ versus TRPC6 alone. (F) Rat smooth muscle cells transfected with rat TRPC6 siRNA. Quantitative RT-PCR for TRPC1-TRPC7 was performed on RNA. Trpc5 and -7 mRNA were not detectable. Percent change of Trpc gene expression in cells transfected with TRPC6 versus control siRNA is shown. (G) Myocytes were cotransfected with RCAN1luciferase and TRPC6 siRNA alone or with ET-1 or PE. Fold activation over RCAN1-luciferase with vehicle alone is shown. Control values were assigned as 1.0.
RCAN1-luciferase and siRNAs for rat TRPC6 in the presence or absence of ET- 1 or PE, an $\alpha$-adrenergic hypertrophic agonist. Addition of TRPC6 siRNA specifically suppressed expression of TRPC6, but not the other TRPC transcripts (Figure 3F). Knockdown of endogenous TRPC6 significantly reduced the inducible expression of RCAN1-luciferase by ET-1 or PE (Figure 3G), suggesting that $G$ protein-coupled receptor agonists activate the calcineurin-NFAT pathway through TRPC6.

Increased expression of TRPC6 induces pathologic bypertrophy in vivo. To investigate the potential consequences of increased cardiac expression of TRPC6 in vivo, we generated $\mathrm{Tg}$ mice expressing rat TRPC6 in a heart-specific manner using the $\alpha$-MHC promoter. Three independent $\mathrm{Tg}$ mouse lines with high, intermediate, and low levels of transgene expression were obtained ( $\mathrm{Tg}$ L23, Tg L16, and $\mathrm{Tg} \mathrm{L8}$, respectively; Figure 4, A-C). Immunocytochemistry using TRPC6 antibody showed that overexpressed TRPC6 protein was preferentially localized to the membrane of ventricular myocytes isolated from Tg hearts (Figure 4D). To confirm that functional TRPC6 channels were properly processed and targeted to the cardiomyocyte cell membrane, we measured cationic current across the membrane using electrophysiologic techniques (Figure 4, $\mathrm{E}$ and $\mathrm{F}$ ). We found that current density was significantly larger in ventricular myocytes isolated from Tg L16 hearts compared with WT littermates. The peak inward current was $3.8 \mathrm{pA} / \mathrm{pF}$ in Tg versus 2.5 $\mathrm{pA} / \mathrm{pF}$ in WT cardiomyocytes, and the peak outward current was 23.3 $\mathrm{pA} / \mathrm{pF}$ in Tg versus $11.7 \mathrm{pA} / \mathrm{pF}$ in WT cardiomyocytes.

To further evaluate the increased expression of functional TRPC6 channels in Tg myocytes, we activated the TRPC receptors by treating cells with ET-1 and performed electrophysiologic recordings (Figure 4G). In these experiments, ET-1-induced increases in both inward and outward currents in 

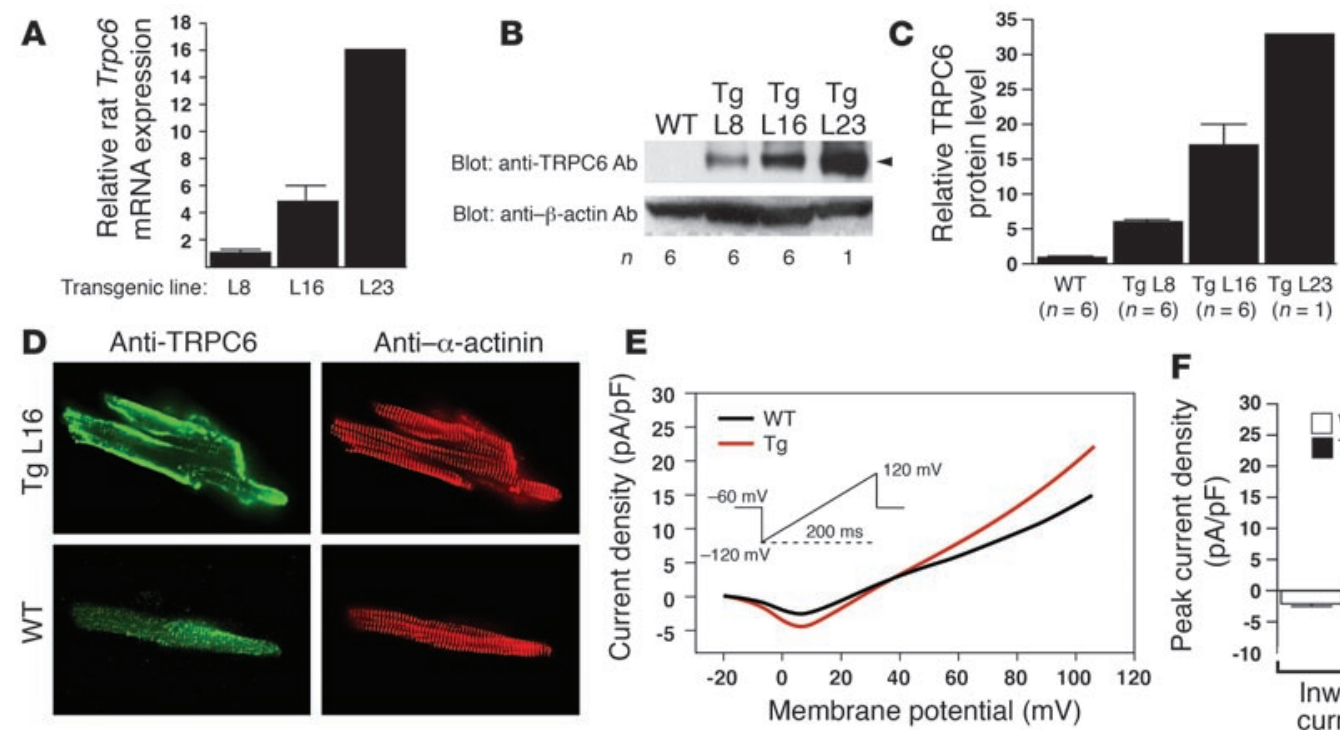

G

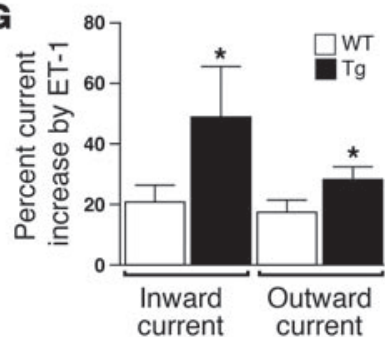

H

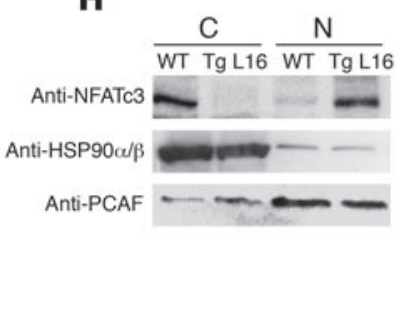

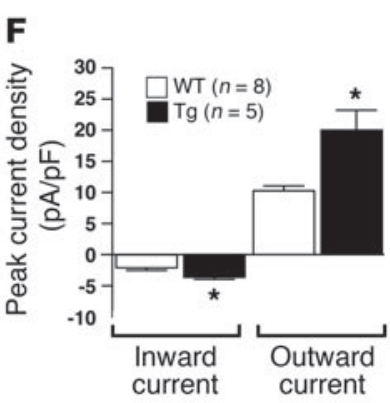

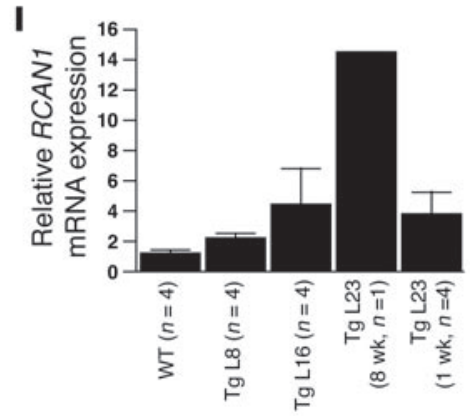

Figure 4

Expression of TRPC6 in hearts of Tg mice. (A) Rat Trpc6 mRNA levels, shown relative to Tg L8 mice and normalized by $18 \mathrm{~S}$ RNA levels, were determined using RT-PCR. (B) Western blot analysis for TRPC6 and $\beta$-actin expression in hearts. (C) Relative TRPC6 protein expression in WT (assigned as 1.0) and TRPC6 Tg mice, determined by densitometry. (D) Immunocytochemistry of adult myocytes isolated from TRPC6 Tg L16 (12 wk) and WT littermates using TRPC6 antibody. Green, anti-TRPC6; red, anti- $\alpha$-actinin. Magnification, $\times 400$. (E) TRPC current was measured by electrophysiologic voltage-clamp in ventricular myocytes isolated from Tg L16 and WT hearts. Current-voltage relations revealed increased current density in Tg L16 myocytes, consistent with increased functional TRPC channel expression at the cell surface. Voltage ramp protocol imposed on cells is inset. (F) Mean values of peak TRPC current density recorded in Tg L16 versus WT myocytes. ${ }^{*} P<0.05$. (G) Mean values of percent TRPC6 current increase on exposure to $10 \mathrm{nM}$ ET-1. (H) Cytoplasmic $(\mathrm{C})$ and nuclear $(\mathrm{N})$ proteins immunoblotted with anti-NFATc3, anti-PCAF (nuclear marker), or anti-HSP90 $\alpha / \beta$ (cytoplasmic marker). (I) RCAN1 (exon 4) mRNA levels were determined by realtime RT-PCR using RNA from hearts of 7- to 8-week-old WT, Tg L8, Tg L16, and Tg L23 mice. mRNA levels relative to RCAN1 mRNA in WT (assigned as 1.0) normalized by 18S RNA are shown.

Tg cardiomyocytes were significantly greater than in WT cardiomyocytes. These data are consistent with increased expression of functional TRPC6 channels in Tg cardiomyocytes.

Further evidence that functional TRPC6 channels are expressed in Tg mice was provided by the observation that NFATc3 accumulated in the nucleus (Figure 4H) and was hypophosphorylated (data not shown) in TRPC6 Tg L16 ventricles compared with ventricles of WT littermates. Consistent with these findings, the expression of RCAN1 (exon 4) mRNA, a sensitive marker of calcineurin signaling, was significantly increased (Figure 4I), even in Tg L8, the mouse line with the lowest expression of TRPC6. The majority of mice derived from the high-expressing line Tg L23 died 5-12 days after birth (Figure 5A) and exhibited increased heart weight/body weight $(\mathrm{HW} / \mathrm{BW})$ ratios at 7 days after birth (Figure 5, B-D). Histologic examination of $\alpha$-MHC-TRPC6 Tg hearts revealed enlarged atria and ventricles with heterogeneity of myocyte size (Figure 5E), suggesting severe cardiomyopathy as the cause of death. In contrast, Tg L16 mice, with intermediate TRPC6 expression, showed no increase in HW/BW ratios at 8 weeks of age, but they developed cardiomegaly and congestive heart failure around 30 weeks of age (Figure 5, F-H). Histologic analyses of these mice revealed cardiac dilatation with heterogeneity of myocyte size and interstitial fibrosis as well as congestion of the lungs, indicative of heart failure (Figure 5, I and J). We conclude that increased expression of TRPC6 in the heart can evoke pathologic cardiac remodeling leading to cardiomyopathy in vivo.

Tg L8, the mouse line with the lowest expression of TRPC6, showed no difference in HW/BW ratios compared with WT littermates until at least 20 weeks of age (Figure 6A). Echocardiography also demonstrated no significant difference in ventricular wall thickness or systolic function between these Tg mice and WT littermates (Table 1). To determine whether expression of TRPC6 
A

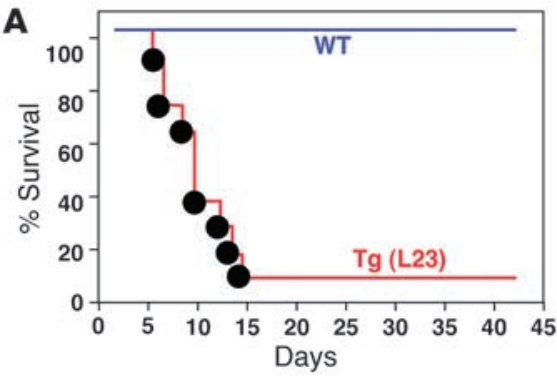

E

E WT
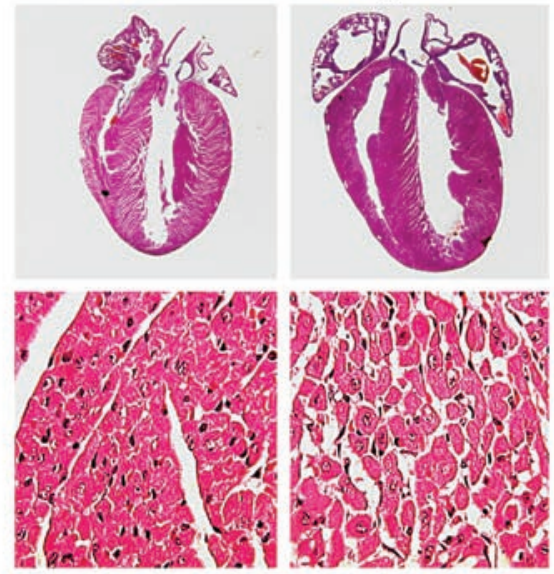

Tg L23

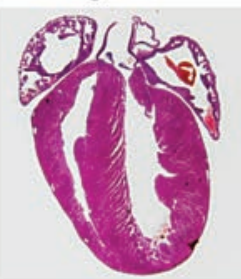

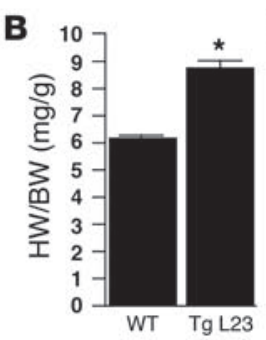

F

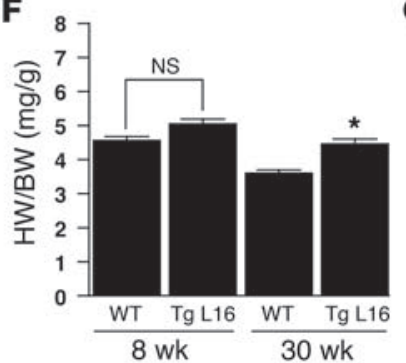

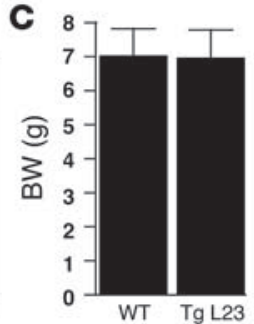

D

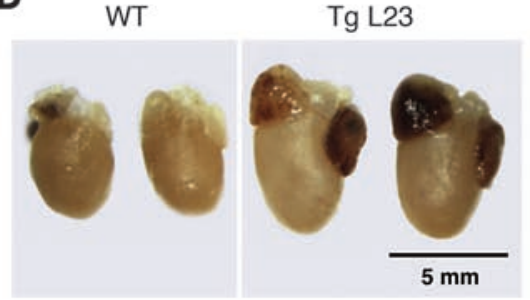

I

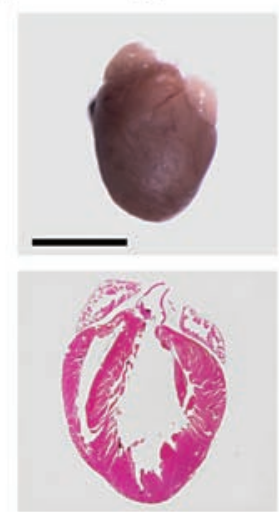

$\operatorname{Tg} \mathrm{L} 16$
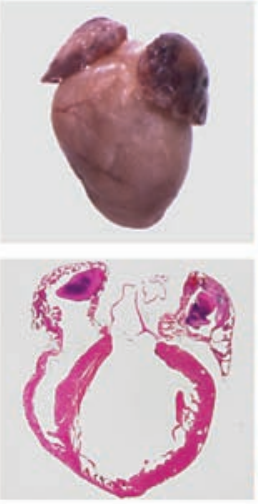

G

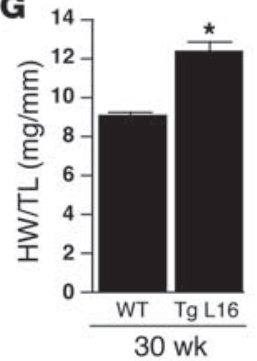

H WT

Tg L16

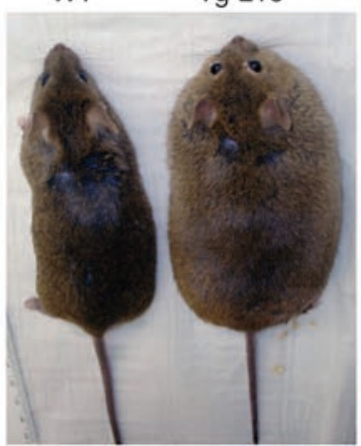

WT

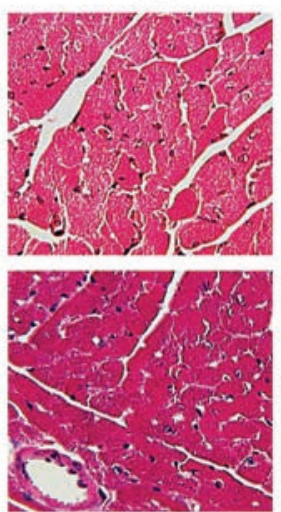

Tg L16

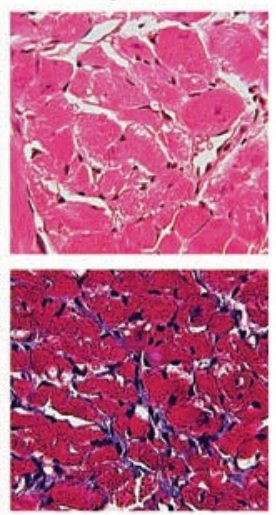

J

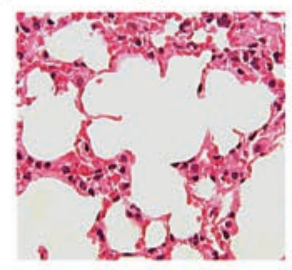

$\mathrm{Tg} \mathrm{L16}$

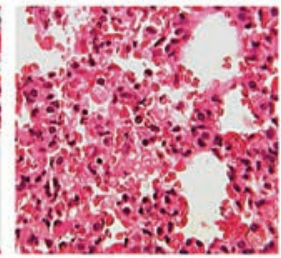

\section{Figure 5}

Cardiomyopathy in TRPC6 Tg mice. (A) Kaplan-Meier survival analysis of TRPC6 Tg L23 ( $n=10$ per group). (B and C) HW/BW ratios (B) and BW (C) in WT and TRPC6 Tg L23 mice ( $n=6$ per group). (D) Gross hearts of WT and TRPC6 Tg L23 mice at 7 days of age. (E) Histologic analysis of hearts from TRPC6 Tg L23 and WT mice at 7 days of age. Hearts were sectioned longitudinally and stained with H\&E. (F) HW/BW ratios in WT and TRPC6 Tg L16 mice ( $n=3$ per group). (G) HW/tibia length (HW/TL) ratios in WT and TRPC6 Tg L16 mice at 30 weeks of age ( $n=3$ per group). (H) TRPC6 Tg L16 and WT mice at 30 weeks of age. (I) Histologic analysis of hearts from TRPC6 Tg L16 and WT mice at 30 weeks of age. Hearts were sectioned longitudinally and stained with H\&E or Masson's trichrome (bottom panels). Scale bar: $5 \mathrm{~mm}$. (J) Histologic analysis of lungs from TRPC6 Tg L16 and WT mice at 30 weeks of age. Lungs were sectioned longitudinally and stained with H\&E. * $P<0.05$ versus WT. Magnification, $\times 40(\mathbf{E}$, bottom panels; I, right panels; and $\mathbf{J})$.

sensitizes the heart to stress, we subjected these animals to pressure overload by TAB and found that they exhibited an exaggerated response to pressure overload, with a dramatic increase in HW/BW ratios and decreased systolic function compared with WT littermates (Figure 6, B and C, and Table 1). These results indicate that increased expression of TRPC6 sensitizes the heart to pathologic hypertrophic signaling, leading to cardiac dysfunction.
Regulation of $\beta-M H C$ expression by the TRPC6-calcineurin-NFAT pathway. Although mouse line $\mathrm{Tg} \mathrm{L} 8$ showed no obvious hypertrophic phenotype until 20 weeks of age in the absence of stress, $\beta$ - $M H C$ mRNA expression was significantly increased and brain natriuretic peptide $(B N P)$ mRNA expression was moderately increased, while expression of ANP, skeletal $\alpha$-actin, $\alpha$-MHC, and sarco/endoplasmic reticulum $\mathrm{Ca}^{2+}$-ATPase isoform2 (SERCA2) was not signifi- 

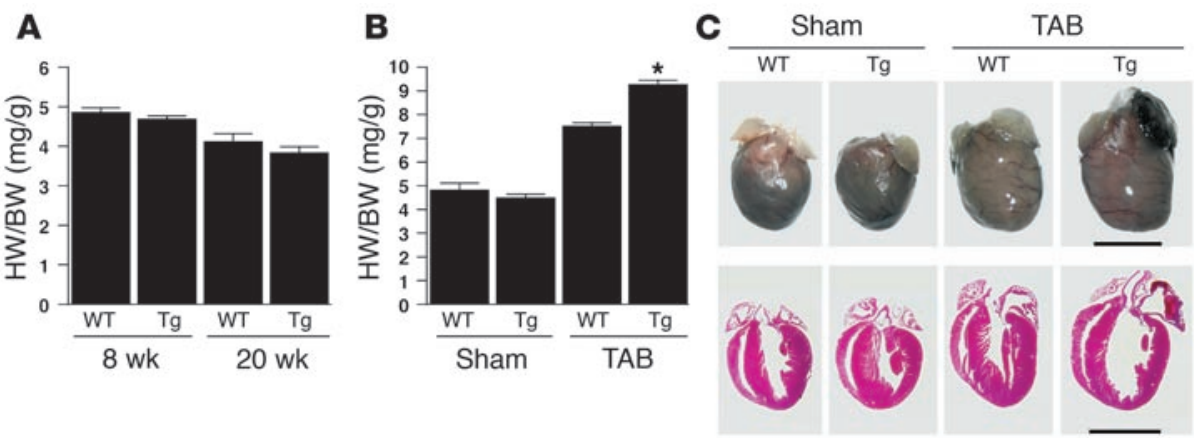

Figure 6

Accelerated pathologic remodeling in response to TAB in TRP6 Tg L8 mice. (A) HW/BW ratios in WT and TRPC6 Tg L8 mice at 8 and 20 weeks of age ( $n=6$ per group) and (B) subjected to TAB ( $n=3$ per group). ${ }^{*} P<0.05$ versus WT. (C) Gross hearts of WT and TRPC6 Tg L8 mice after 3 weeks of TAB. Hearts were sectioned longitudinally and stained with H\&E. Scale bars: $5 \mathrm{~mm}$. nificantly contributes to the inducible expression of the $\beta$-MHC gene in the cardiac hypertrophic response.

\section{Discussion}

The calcium/calmodulin-dependent serine/threonine protein phosphatase calcineurin acts as a key regulator of hypertrophic signaling through its downstream transcriptional effector NFAT, which is directly dephosphorylated by calcineurin and causes nuclear translocation and activation of NFATdependent gene transcription $(12,34)$. However, the upstream source of calcium responsible for activation of the calcineurin-NFAT pathway has not been fully defined. The results of the cantly altered in the hearts of these mice (Figure 7A). These results suggest that signaling pathways downstream of TRPC6 specifically control $\beta-M H C$ gene expression. Indeed, $\beta-M H C$ mRNA expression correlated with the level of TRPC6 transgene expression in the different lines of $\alpha$-MHC-TRPC6 Tg mice (Figure 7B). These results were corroborated by real-time RT-PCR, which showed $\beta-M H C$ mRNA expression to be upregulated compared with ANP and BNP in hearts of $\alpha$-MHC-calcineurin Tg mice at different ages (Figure $7, \mathrm{C}$ and $\mathrm{D}$ ). These results suggest that TRPC6-calcineurin-NFAT signaling preferentially regulates $\beta-M H C$ gene expression.

To explore the mechanistic basis of the sensitivity of the $\beta-M H C$ gene to calcineurin-NFAT signaling, we fused a $-396 \mathrm{bp}$ promoter region of the mouse $\beta-M H C$ gene to a luciferase reporter $(-396 \beta$ MHC-luc) (30). This promoter region contains multiple MCATbinding sites, 1 GATA site, and 1 NFAT site $(31,32)$ (Figure $8 \mathrm{~A})$. The GATA and NFAT sites are conserved in $\beta$ - $M H C$ promoters of different mammalian species, and GATA and NFAT factors have been reported to bind to these sites $(31,32)$. TRPC6, as well as constitutively active NFAT3 (NFATc4 $\Delta 317)$ and $\mathrm{CnA} \Delta \mathrm{C}$, activated the $-396 \beta$-MHC-luc reporter in cardiomyocytes (Figure $8 \mathrm{~B}$ ). The stimulatory activity of TRPC 6 or NFATc $4 \Delta 317$ was reduced when either the GATA or NFAT sites in the promoter were mutated. In addition, double mutation of both GATA and NFAT sites abolished responsiveness to TRPC6 or NFATc4, demonstrating that the TRPC6-calcineurin-NFAT pathway directly activates $\beta$ - $M H C$ gene transcription through these sites (Figure 8C). TRPC6 expression also enhanced ET-1-inducible activation of -396 3 -MHC-luc (Figure 8D). The TRPC6-, ET-1-, and PE-induced activation of -396 3 -MHC-luc was significantly inhibited in the presence of calcineurin inhibitors cyclosporin A (Figure 8, E and G) and RCAN1 (Figure 8, $\mathrm{F}$ and $\mathrm{H}$ ), indicating that the calcineurin-NFAT pathway is involved in -3963-MHC-luc activation by multiple hypertrophic stimuli (Figure 8, E-H). Furthermore, the increase in $\beta-M H C$ gene expression in TRPC6 Tg L16 mice was significantly attenuated in double Tg mice expressing both TRPC6 and the calcineurin inhibitor RCAN1 in the heart (Figure 8I) (33).

To examine the role of endogenous TRPC6 in hypertrophic stimuli-inducible $\beta-M H C$ gene transcription, cardiomyocytes were cotransfected with -3963-MHC-luc and siRNAs for rat TRPC6 in the presence or absence of PE or ET-1. As shown in Figure 8J, TRPC6 knockdown reduced the induction of $-396 \beta$-MHC-luc activity by PE or ET-1. Collectively, these findings indicate that TRPC6 sig- present study reveal a key contribution of TRPC family channels to activation of the calcineurin-NFAT pathway during pathologic cardiac remodeling. The following observations support this conclusion: (a) Expression of TRPC6, a mediator of receptor-activated $\mathrm{Ca}^{2+}$ entry in smooth muscle cells (35-37), was increased in the hearts of mice expressing activated calcineurin and in response to TAB as well as in failing human hearts and primary cardiomyocytes stimulated with ET-1; (b) The promoter of the TRPC6 gene contains 2 conserved response to calcineurin-NFAT signaling; (c) TRPC6 overexpression in cardiomyocytes activated the calcineurin-NFAT-dependent RCAN1 promoter in vitro and induced expression of the endogenous RCAN1 gene in vivo. (d) Cardiac overexpression of TRPC6 in Tg mice caused exaggerated pathologic cardiac remodeling under basal and stressed conditions that depended on the Trpc6 transgene dosage. Together, these results indicate that TRPC6 forms a positive regulatory circuit in the calcineurin-NFAT pathway, facilitating the transition to cardiomyopathy. A model to account for these results is shown in Figure 9.

A regulatory circuit of TRPC6-calcineurin-NFAT in the bypertrophic heart. The association of SOCE with NFAT signaling has been well documented in nonexcitable cell types $(20,21,38)$. However, relatively little is known of the potential roles of SOCE in excitable cells. Recently, TRPC3 expression was reported to be upregulated in skeletal muscle by neuromuscular activity in a calcineurin-dependent manner, thereby providing a mechanism for modulation of muscle fiber type (26). Hypertrophic hearts isolated from $\alpha$-MHC-calcineurin $\mathrm{Tg}$ mice showed increased expression of Trpc6 mRNA. SOCE has been detected in embryonic and neonatal cardiomyocytes (11) and

\section{Table 1}

Echocardiographic analysis of TRPC6 Tg L8 and WT mice subjected to TAB

\begin{tabular}{lcccc}
\hline & HR $(\mathbf{b p m})$ & LVDd $(\mathbf{m m})$ & LVDs $(\mathbf{m m})$ & EF $(\%)$ \\
WT sham & $690 \pm 30$ & $3.67 \pm 0.32$ & $1.59 \pm 0.06$ & $91.0 \pm 1.4$ \\
WT TAB & $740 \pm 20$ & $4.17 \pm 0.23$ & $2.26 \pm 0.58$ & $80.1 \pm 9.1$ \\
Tg L8 sham & $720 \pm 20$ & $3.85 \pm 0.25$ & $1.74 \pm 0.72$ & $87.5 \pm 10.0$ \\
Tg L8 TAB & $660 \pm 30$ & $4.64 \pm 0.24$ & $3.64 \pm 0.29$ & $46.2 \pm 7.8^{A}$
\end{tabular}

${ }^{A} P<0.05$ versus WT TAB. Values are mean \pm SEM. HR, heart rate; LVDd, left ventricular end diastolic dimension; LVDs, left ventricular end systolic dimension; EF, ejection fraction. NFAT sites, which were required for activation of the promoter in 

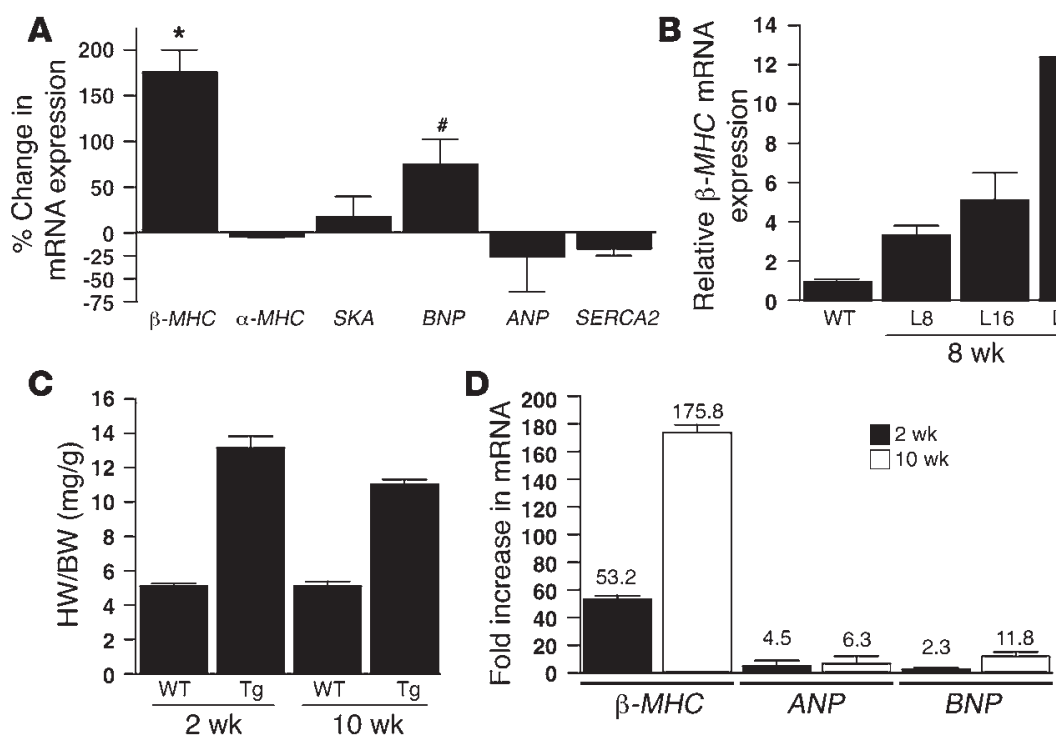

Figure 7

Induction of $\beta-M H C$ gene expression in TRPC6 Tg hearts. (A) Expression of cardiac genes $\beta-M H C, B N P, \alpha-M H C, A N P, \alpha$-skeletal actin (SKA), and SERCA2 in hearts isolated from in TRPC6 $\mathrm{Tg} L 8$, the lowest-expressing line. Percent change in relative mRNA levels normalized by $18 \mathrm{~S}$ RNA levels of Tg mouse hearts compared with WT littermates is shown. $n=4$ per group. ${ }^{*} P<0.001$, $\# P<0.05$ versus WT. (B) Expression of $\beta-M H C$ gene in hearts of different Tg TRPC6 mouse lines. Shown are $\beta-M H C$ mRNA levels relative to WT (assigned as 1.0) normalized by $18 S$ RNA levels. $n=4$ per group, except for 8-wk Tg L23 $(n=1)$. (C) HW/BW ratios of WT and calcineurin Tg mice at 2 and 10 weeks of age. $n=4$ per group. (D) Expression of $\beta-M H C, A N P$, and $B N P$ in calcineurin $\mathrm{Tg}$ mice at 2 and 10 weeks of age. Fold increase in mRNA levels over WT (assigned as 1.0) normalized by $18 \mathrm{~S}$ RNA levels is shown. $n=4$ in each group.

contributes to NFAT activation in neonatal cardiomyocytes (10). In contrast, SOCE was reported to be reduced in adult cardiomyocytes, suggesting that it contributes only marginally to cardiac function under normal physiologic conditions (11). These previous findings are consistent with the relatively low level of NFAT activity in the normal adult heart compared with the neonatal heart (39). Pathologic stimuli cause a delayed and sustained increase in NFAT activity (39). Our findings show that TRPC6 was upregulated in an NFATdependent manner during pathologic remodeling of the heart, revealing a positive amplification circuit for NFAT signaling.

TRPC6 is an essential component of receptor-activated cation channels. In smooth muscle, TRPC6-mediated cation influx triggers membrane depolarization and consequent activation of L-type $\mathrm{Ca}^{2+}$ channels, leading to $\mathrm{Ca}^{2+}$ influx and smooth muscle contraction (35-37). In addition, TRPC6 has been demonstrated to be involved in the proliferation of smooth muscle cells by increasing cytoplasmic $\mathrm{Ca}^{2+}$ concentration and activating subsequent signaling pathways. TRPC6 is abundantly expressed in the pulmonary system, and TRPC6 gene expression is increased in pulmonary artery smooth muscle cells (PASMCs) in response to idiopathic pulmonary arterial hypertension (IPAH). Intriguingly, bosentan, a dual endothelin receptor blocker that has been used clinically to treat IPAH, reduces the increase of TRPC6 expression in PASMCs (40). These findings suggest that an ET-1-TRPC6-calcineurin-NFAT circuit exists in PASMCs as well as in cardiomyocytes.

NFAT-independent pathways have also been shown to activate the Trpc6 gene. PDGF has been shown to stimulate Trpc6 gene expression via c-Jun- and STAT3-dependent pathways, which are also known to be involved in cardiac hypertrophy (41). Thus, both NFAT-dependent and -independent pathways may cooperatively activate TRPC6 gene transcription during cardiac remodeling.

Unexpectedly, mice deficient in TRPC6 showed increased agonistinduced contractility of aortic rings and elevated blood pressure accompanied by increased expression of TRPC 3 (42), a constitutively active TRPC. This resulted in enhanced basal and agonist-induced cation entry into smooth muscle cells and led to greater depolarized membrane potentials and increased smooth muscle contractility. Knockdown of TRPC3 using siRNA abolished the higher basal cation entry observed in TRPC6 $6^{-/-}$smooth muscle cells. Further evaluation of these mutant mice is necessary to define the precise role of TRPC6 in the cardiovascular system.

Recently, novel proteins Orai1 (CRACM1) and STIM1 have been shown to functionally interact and regulate SOCE (43-48). STIM1 interacts with TRPCs to enhance SOCE (49). These results imply that a multiprotein complex containing Orai1, STIM1, and TRPCs regulates SOCE function. Analysis of the expression and function of Orai1 and STIM1 in cardiac hypertrophy may provide insights into the mechanisms involved in activation of the calcineurin-NFAT pathway during cardiac remodeling.

Control of $\beta-M H C$ by the TRPC6-calcineurin-NFAT signaling pathway. Our results revealed that a TRPC6-calcineurin-NFAT pathway regulates $\beta-M H C$ gene expression, a marker for pathologic hypertrophy and heart failure in human and rodents (50-53). In slow skeletal muscle, the calcineurin-NFAT signaling pathway is known to induce $\beta$-MHC expression (54-56), and mutations in NFAT sites in the $\beta-M H C$ promoter decrease transcriptional activity in slow skeletal muscle (57). In this study, we showed that TRPC6 and NFAT activated the $\beta-M H C$ promoter through the GATA and NFAT sites in cardiomyocytes. We have previously shown that GATA and NFAT factors synergistically activate cardiac gene transcription (12). Mutation of an NFAT site in the $\beta$ - $M H C$ promoter causes only a small reduction of the response to PE, suggesting that GATA activation is required for NFAT to fully induce $\beta$ - $M H C$ promoter activity during cardiac hypertrophy and that signaling pathways other than the calcineurin-NFAT pathway are also involved in regulation of the $\beta-M H C$ gene (31). In this regard, local $\mathrm{Ca}^{2+}$ release from the type 2 inositol-1,4,5-triphosphate (IP3) receptor, which is mainly located in the nuclear envelope, activates $\mathrm{Ca}^{2+} /$ calmodulin-dependent protein kinase II (CaMKII) and hypertrophic gene transcription in adult ventricular myocytes (58). In most noncardiac cells, $\mathrm{Ca}^{2+}$ release from the IP3 receptor is associated with SOCE (22). Other $\mathrm{Ca}^{2+}$-dependent signaling, such as that of CaMKII, may also be involved in the TRPC6-mediated signaling pathways. Our present data showing that the TRPC6-, ET-1-, or PE-induced activation of the $\beta$-MHC promoter was inhibited in the presence of calcineu- 
A

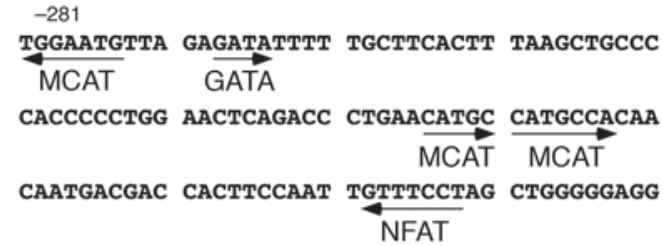

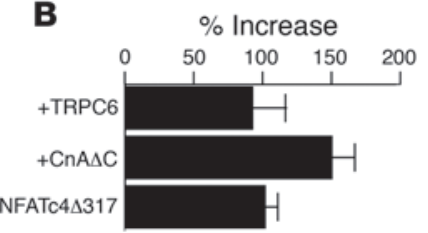

AGGGG -157

C

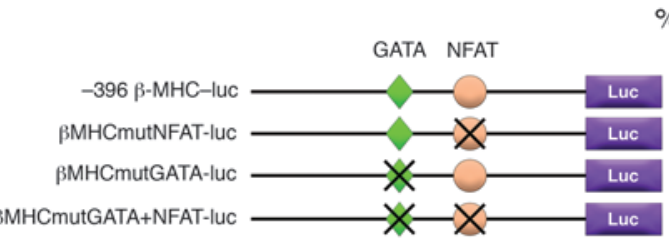

$\%$ Increase by NFATc $4 \Delta 317$

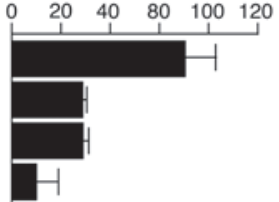

$\%$ Change by TRPC6
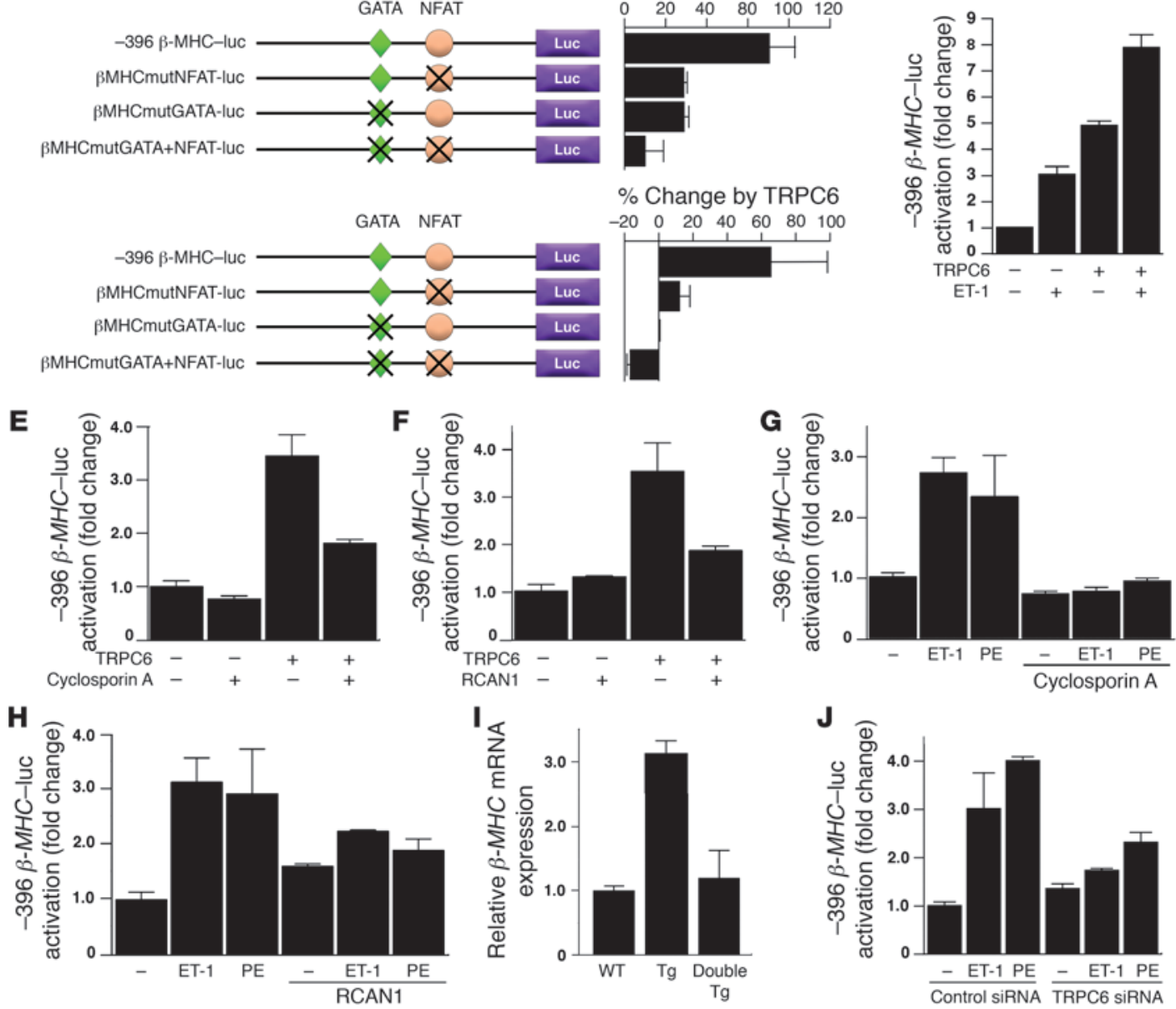

Figure 8

The TRPC6-calcinerurin-NFAT pathway directly regulates $\beta-M H C$ gene transcription. (A) Sequence of the mouse $\beta-M H C$ promoter. MCAT, NFAT, and GATA binding sites are shown. (B and C) Rat neonatal ventricular myocytes were cotransfected with (B) $-396 \beta-M H C-l u c$ and an expression plasmid of TRPC6, CnA $\Delta$ C, or NFATc4 $\Delta 317$ or (C) $-396 \beta-M H C-l u c$ with NFAT or GATA mutants and an expression plasmid encoding NFATc4 $\triangle 317$ or TRPC6 (bar graphs show percent increase or change in luciferase activity by NFATc4 317 or rat TRPC6 compared with control vector); (D-F) -396 $\beta-M H C-l u c$ and an expression vector encoding TRPC6 in the presence or absence of (D) ET-1, (E) cyclosporin A, or $(\mathbf{F})$ an expression plasmid of RCAN1; (G, H, and $\mathbf{J})-396 \beta-M H C-l u c$ in the presence or absence of ET-1, PE, or (G) cyclosporin A, (H) an expression vector encoding RCAN1, or (J) control or TRPC6 siRNAs. (I) Relative expression of $\beta$-MHC mRNA was measured using real-time RT-PCR in hearts of WT, $\beta$-MHC-TRPC6 (Tg), and $\beta$-MHC-TRPC6; $\beta$-MHC-RCAN1 (double Tg) mice. Fold change over control -396 $\beta-M H C-$ luc activity (assigned as 1.0) is shown.

rin inhibitors cyclosporin A or RCAN1 and that the knockdown of TRPC 6 reduced the response of the $\beta-M H C$ promoter to ET- 1 and PE in cardiomyocytes strongly supports the conclusion that TRPC6 forms a positive regulatory circuit with the calcineurin-NFAT pathway during cardiac hypertrophy that is accompanied by the induction of $\beta-M H C$ gene expression.

$\alpha$-MHC-calcineurin Tg mice show a dramatic increase in $\beta-M H C$ mRNA expression in the postnatal heart (12). In most mouse mod- els of cardiac hypertrophy, ANP appears to be the earliest and most sensitive marker $(59,60)$, although some severe cardiomyopathic mice show a higher increase in $\beta-M H C$ than Anp gene expression (61). Inhibition of the calcineurin-NFAT pathway by overexpression of RCAN1 or the NFAT inhibitor GSK3 $\beta$ results in selective repression of $\beta-M H C$ gene expression in mouse models of myocardial infarction or calcineurin $\mathrm{Tg}$ mice, respectively, supporting the notion of a preferential contribution of the calcineurin-NFAT 


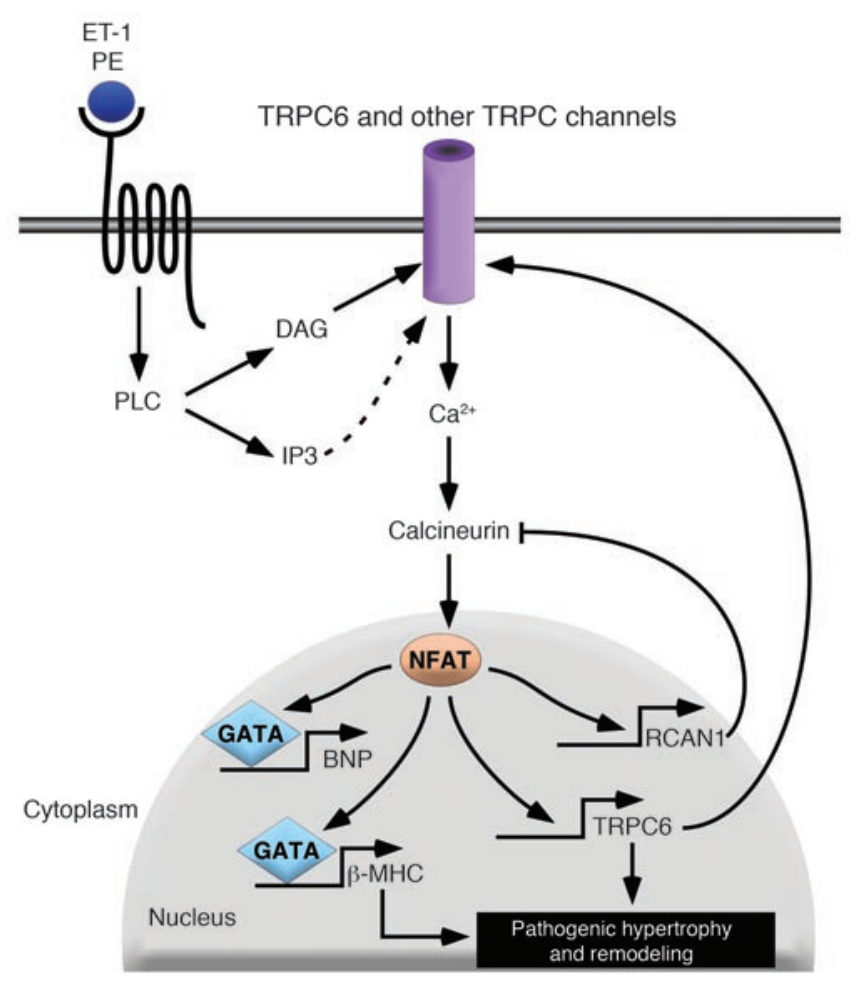

pathway in regulation of $\beta-M H C$ gene expression in the heart (62, 63 ). The finding that forced overexpression of the $\beta-M H C$ gene in the mouse heart led to a significant decrease in systolic function $(64,65)$ suggests that increased expression of $\beta$-MHC in cardiac hypertrophy may be a maladaptive response leading to cardiomyopathy. In this regard, mutations in the $\beta-M H C$ gene cause human hypertrophic cardiomyopathy and dilated cardiomyopathy, implying that proper expression of $\beta-\mathrm{MHC}$ is required for maintenance of normal cardiac structure and function $(66,67)$. Thus, induction in $\beta$-MHC gene expression may be one of the downstream mechanisms by which the TRPC6-calcineurin-NFAT pathway induces pathologic cardiac remodeling.

TRPC6 as a potential therapeutic target. In addition to the evidence implying that a shift of MHC isoforms from $\alpha-\mathrm{MHC}$ to $\beta$-MHC observed in cardiomyopathy may be more than just a marker for the severity of the disease state and might actually contribute to its development $(50-53,64,65)$, it has previously been shown that the improvement of left ventricular function is associated with a coordinate increase in $\beta-M H C$ and a decrease in $\alpha-M H C$ mRNA expression in patients with idiopathic dilated cardiomyopathy $(50,52)$. Indeed, cardiac overexpression of $\alpha$-MHC in rabbits confers cardioprotection to ventricular pacing (68). Thus, the results of this study showing the involvement of TRPC6-calcineurin-NFAT signaling in the regulation of $\beta-M H C$ gene transcription during pathologic cardiac remodeling point to the control of $\beta-M H C$ expression as a possible therapeutic target for the treatment of heart failure and suggest that TRPC6 receptor antagonists might serve to normalize cardiac gene expression and contractility in the setting of pathologic cardiac remodeling.

\section{Methods}

Plasmids. A DNA fragment from the 5 ' flanking region of the mouse $\beta$-MHC gene spanning bp -396 to $\mathrm{bp}+96$ relative to the transcription start site was

\section{Figure 9}

Proposed model of the role of TRPC6 in the calcineurin-NFAT pathway in vivo. TRPC6 gene transcription is activated by the calcineurin-NFAT pathway, and an increase in the expression of TRPC6 further activates the calcineurin-NFAT pathway. The $\beta-M H C$ gene is one of the downstream targets of this pathway, and its expression may alter cardiac contractility. Activation of BNP expression also provides a negative feedback loop to curtail hypertrophic signaling. TRPC6 forms a positive regulatory circuit in calcineurin-NFAT pathways leading to pathologic remodeling. DAG, diacylglycerol; GATA, GATA-binding protein; IP3, inositol-1,4,5-triphosphate; PLC, phospholipase C.

isolated by PCR using mouse genomic DNA as a template. The fragment was inserted upstream of a luciferase gene in the pGL3 vector (Promega) using Xhol/HindIII sites. The following primers were used: sense, 5'-TGAACCGCTCGAGAAGGCTTCTAAGGGG-3'; antisense, 5'-ATGCCCAAGCTTACTTCCTGAACCTGGAG-3'. To generate mutations in the GATA and NFAT sites of the $\beta$-MHC 5 ' flanking region, we used a PCR-based mutagenesis kit (QuikChange; Stratagene) with the following primers: mutGATA sense, 5'-GAATGTTAGAGGCGTTTTTGCTTC-3'; mutGATA antisense, 5'-GAAGCAAAAACGCCTCTAACATTC-3'; mutNFAT sense, 5'-TTCCAATTGTGGGCTAGCTGGGG-3', mutNFAT antisense, 5'-CCCCAGCTAGCCCACAATTGGAA- ${ }^{\prime}$. All vectors were verified by sequencing.

A 913-bp fragment containing the upstream region and the 5' untranslated region of the mouse Trpc6 gene was isolated by PCR using mouse genomic DNA as a template, and the fragment was inserted upstream of a luciferase gene in the pGL3 vector using XhoI/HindIII sites (-913TRPC6luc). The following primers were used: sense, $5^{\prime}$-TCCGCTCGAGCGGGTTGAGAAGGTTTTGATTTTGACAGAT-3'; antisense, 5'-ACCCAAGCTTGGGCACAGTGCCTGGCCGGCCTGCACC- 3 '. To generate the deletion mutant of the Trpc6 5' flanking region from -617 to the ATG (-617TRPC6luc), the -913TRPC6-luc construct was digested with XhoI and BglII to excise nucleotide bps from -913 to -617 and then blunted and ligated using a Blunting kit (Takara). To generate mutations in either the distal or proximal NFAT sites (mutNFAT1 or mutNFAT2, respectively) of -913TRPC6-luc, PCR-based mutagenesis was performed using the following primers; mutNFAT1 sense, 5'-AGGGAGGATTGCGATTAAAGCAAGCA-3'; mutNFAT1 antisense, $5^{\prime}$-TGCTTGCTTTAATCGCAATCCTCCCT-3'; mutNFAT2 sense, 5'-GCAGACACCATCTCGCAATGCCCTTTTT-3'; mutNFAT2 antisense, 5'-AAAAAGGGCATTGCGAGATGGTGTCTGC-3'.

RCAN1-luciferase in which RCAN1 intron 3 containing 15 NFAT sites was inserted upstream of the luciferase reporter gene was kindly provided by B. Rothermel (University of Texas Southwestern Medical Center; ref. 29). A pcDNA3-based expression vector for rat TRPC6 was kindly provided by E. Satoh (Aomori University, Aomori, Japan).

Cell culture and transfection. Primary neonatal rat ventricular myocytes were isolated and grown as described previously (12). Twenty-four hours after plating, the myocytes were transfected with $200 \mathrm{ng}$ of reporter plasmid and $200 \mathrm{ng}$ of expression vectors for 12 hours using GeneJammer (Stratagene), unless indicated otherwise. A RSV-driven lacZ expression vector was included in all transfections as an internal control. After transfection, serum was removed from the growth medium. Six hours after serum deprivation, ET-1 (10 nM), PE (100 mM), or vehicle was added, and the cells were maintained for an additional 48 hours.

Luciferase assay. Cells were harvested, and luciferase and control $\beta$-galactosidase activities were measured according to the manufacturer's instructions (Promega). All assays were performed at least twice with triplicates for each assay.

Gel mobility shift assay. Gel mobility shift assay was performed with doublestranded oligonucleotides corresponding to consensus NFAT or TRPC6 
NFAT-like sequences. Sequences of the probes were as follows: NFAT consensus, 5'-CTAGGAAAATTCACTAGGAAAATTCA-3'; TRPC6 NFAT1, 5'-AGGATTTTCCTTAAGCAGGATTTTCCTTAAAGC-3'; TRPC6 NFAT2, 5'-ACCATCGGAAAATGCCCACCATCGGAAAATGCCC-3'. For gel mobility shift assay utilizing the NFATc $4 \Delta 317$-containing Rel homology domain, $2 \mu \mathrm{l}$ of a coupled in vitro translation reaction (TNT kit; Promega) was incubated with ${ }^{32} \mathrm{P}$-labeled oligonucleotide probe (Figure $2 \mathrm{~B}$ ) in the presence of $1 \mu \mathrm{l}$ of poly dIdC $(1.5 \mathrm{mg} / \mathrm{ml})$ for 20 minutes at room temperature, followed by nondenaturing electrophoresis. Unlabeled competitor oligonucleotides were added at a 100 -fold molar excess and $2 \mu$ of NFATc4-specific antibody (Calbiochem) was added for supershift experiments. The gel mobility shift assay buffers and electrophoresis conditions were described previously (12).

RNA interference. For RNA interference analysis of TRPC6, a siGENOME SMART pool reagent against rat TRPC6 (M-095094-00) with guaranteed minimum 75\% mRNA knockdown was purchased from Dharmacon. BLOCK-iT Fluorescent Oligo (Invitrogen) was used as a nonspecific control. For luciferase assays, transfections using 100 pmol siRNAs and 500 ng luciferase reporter plasmids were performed in neonatal rat ventricular myocytes in DMEM with $10 \%$ fetal bovine serum using FuGENE (Roche Applied Science). A RSV-lacZ expression plasmid was included in all transfections as an internal control. Luciferase assay was performed 48 hours after transfection. To verify the efficiency of siRNA-mediated knockdown of TRPC6 expression, rat smooth muscle cells maintained in 6-well dishes were transfected with 200 pmol siRNAs. Cells were harvested 48 hours after transfection for real-time RT-PCR analysis. Using rat TRPC6 siRNA, we observed 77\% reduced expression of endogenous TRPC6 mRNA in rat smooth muscle cells compared with control siRNA. The expression of other TRPC family genes was not significantly changed with the rat TRPC6 siRNA.

Generation of Tg mice. Tg mice expressing a rat TRPC 6 CDNA driven by the cardiac-specific $\alpha$-MHC promoter were generated by standard procedures. $\mathrm{Tg}$ mice carrying the exon 4 splice variant of the human RCAN1 gene driven by the $\alpha$-MHC promoter were kindly provided by B.A. Rothermel (University of Texas Southwestern Medical Center; ref. 33). All animal protocols used in this study were approved by the University of Texas Southwestern Institutional Animal Care and Use Committee.

Western blot analysis. Hearts were homogenized in lysis buffer (Cell Signaling Technology), and $100 \mu \mathrm{g}$ proteins was resolved on a sodium dodecyl sulfate-polyacrylamide gel, transferred to PVDF membrane (Bio-Rad), and immunoblotted with rabbit polyclonal anti-TRPC6 that recognizes both rat and mouse TRPC6 (Alomone Labs). Bands were visualized using antirabbit IgG conjugated to horseradish peroxidase and Western Blotting Luminol Reagent (Santa Cruz Biotechnology Inc.). Densitometry was performed on X-ray film using Scion Image software version 1.62c.

To analyze the phosphorylation state of NFAT, $3 \mathrm{mg} \mathrm{LV}$ extract was immunoprecipitated with anti-NFATc3 antibody (sc-8321; Santa Cruz Biotechnology Inc.) and subjected to Western blot analysis with an antiphosphoserine antibody (61-8100; Zymed) as described previously (54).

In cellular fractionation experiments, nuclear and cytoplasmic extracts were collected as described previously (26). Briefly, to analyze the cellular localization of NFAT, hearts were homogenized with lysis buffer $(20 \mathrm{mM}$ HEPES, pH 7.4; 10 mM NaCl; 1.5 mM MgCl $2 ; 20 \%$ glycerol; 0.1\% Triton-X; $1 \mathrm{mM}$ DTT; and protease inhibitors). Cell suspensions were centrifuged at $600 \mathrm{~g}$ at $4^{\circ} \mathrm{C}$. Supernatants contained the cytosolic fraction, while pellets, the nuclear fraction, were resuspended in lysis buffer supplemented with $500 \mathrm{mM} \mathrm{NaCl}$ and rotated for 1 hour. The nuclear fraction was then centrifuged at $16,000 \mathrm{~g}$ for 10 minutes at $4^{\circ} \mathrm{C}$. Cytoplasmic and nuclear proteins $(50 \mu \mathrm{g})$ were then analyzed by SDS-PAGE and immunoblotted with anti-NFATc3 antibody, anti-PCAF antibody (nuclear marker, sc-8999; Santa Cruz Biotechnology Inc.) or anti-HSP90 $\alpha / \beta$ antibody (cytoplasmic marker, sc-13119; Santa Cruz Biotechnology Inc.).
Thoracic aortic banding. Male mice (6-8 weeks old) either underwent a sham operation or were subjected to pressure overload induced by TAB as described previously (18), using protocols approved by the University of Texas Southwestern Institutional Animal Care and Use Committee. A constriction with a 27-gauge needle was placed in the transverse aorta between the innominate and left carotid arteries. We have shown previously that constriction to a 27-gauge stenosis induces moderate hypertrophy without clinical signs of heart failure or malignant ventricular arrhythmia (18). The mice were killed 3 weeks after TAB. We confirmed the integrity of TAB by inspection of the surgical constriction and by visualization of marked differences in caliber of the right and left carotid arteries.

Histologic analysis. Histologic analysis was performed as described previously (69). Briefly, excised hearts were rinsed in PBS and incubated in Krebs-Henseleit solution lacking $\mathrm{Ca}^{2+}$. Hearts were fixed in $4 \%$ paraformaldehyde for 12 hours at room temperature. Samples were dehydrated with ethanol, mounted in paraffin, and sectioned at $5 \mathrm{~mm}$ thickness. Sections were then stained with $\mathrm{H} \& \mathrm{E}$ to visualize tissue architecture or with Masson's Trichrome stain.

Immunocytochemistry. Adult ventricular myocytes were isolated from the hearts of TRPC6 Tg L16 mice and WT littermates at 12 weeks of age as described previously (70). Cells attached to laminin-coated coverslips were rinsed with PBS, fixed with $4 \%$ formaldehyde for 10 minutes, and permeabilized with $0.1 \%$ Triton X-100 in PBS for 5 minutes. Cells were blocked for 30 minutes at room temperature in 3\% BSA in PBS. Cells were then incubated with primary antibody diluted in 3\% BSA in PBS for 60 minutes at room temperature. Anti-TRPC6 polyclonal antibody (diluted 1:200; Alaomone Labs), and anti- $\alpha$-actinin monoclonal antibody (clone EA-53, diluted 1:400; Sigma-Aldrich) were used. For control experiments, TRPC6 antibody was preincubated with the respective antigenic peptide (diluted 1:1; Alaomone Labs). Secondary antibodies were anti-mouse/rabbit IgG fluorescein isothiocyanate or Texas Red (Vector Laboratories) used at 1:200 dilution. VECTASHIELD with DAPI (Vector Laboratories) was used for mounting.

To analyze the cellular localization of NFAT in the presence or absence of TRPC6, COS-1 cells grown on coverslips in 6-well dishes were transfected with $1 \mu \mathrm{g}$ of each expression vector encoding for NFATc4-GFP fusion protein and TRPC6 or control empty vector. Forty-eight hours after transfection, the transfected cells were fixed and permeabilized as described above. Cells were incubated with anti-TRPC6 polyclonal antibody and followed by secondary anti-rabbit IgG antibody conjugated with Texas Red.

Echocardiographic analysis. Cardiac function was evaluated by echocardiography on conscious mice using a Hewlett Packard Sonos 5500 Ultrasound system with a $12-\mathrm{MHz}$ transducer, as described previously (69). Briefly, views were taken in planes that approximated the parasternal short-axis view (chordal level) and the apical long-axis view. Left ventricle internal diameters and wall thicknesses were measured (at least 3 cardiac cycles) at end systole and end diastole.

Human DNA. Tissue samples of left ventricles from hearts of unidentified humans diagnosed as having failing or nonfailing hearts were obtained from Myogen. This study involves the use of existing pathologic specimens, and the information was recorded in such a manner that the subjects cannot be identified directly or through identifiers linked to the subjects. The Institutional Review Board at University of Texas Southwestern Medical Center, in accordance with the Department of Health and Human Services, considers this work to meet the criteria of exempt review.

Real-time RT-PCR. Total RNA was isolated from cultured neonatal ventricular myocytes or mouse hearts using TRIzoL (Invitrogen) according to the manufacturer's protocol. Real-time 1-step RT-PCR was performed with 20-100 ng total RNA using TaqMan One-Step RT-PCR Master Mix reagent (Applied Biosystems). TaqMan primers and probes for mouse TRPC1, -3 , $-4,-5$, and -7; BNP; skeletal $\alpha$-actin; $\beta$-MHC; $\alpha$-MHC; and rat and human 
TRPC6 were purchased from Applied Biosystems. The primers and probes for mouse ANP, SERCA2, and TRPC6 were as follows: ANP forward, 5'-GCCATATTGGAGCAAATCCT-3'; reverse, 5-GCAGGTTCTTGAAATCCATCA-3'; probe, 5'-FAM-TGTACAGTGCGGTGTCCAACACAGATTAMRA-3'; SERCA2 forward, 5'-CATCTGCTTGTCCATGTCACTT-3'; reverse, 5'-CGGTGTGATCTGGAAAATGAG-3'; probe, 5'-FAM-TCTTGATCCTCTACGTGGAACCTTTGC-TAMRA-3'; TRPC6 forward, 5'-CGCTGCCACCGTATGG-3'; reverse, 5'-CCGCCGGTGAGTCAGT-3'; probe, 5'-FAM-ACTACCCCAGCTTCCGGGGTAATGAAAACA-TAMRA-3'.

Electrophysiologic recordings. Mouse left ventricular cardiomyocytes were isolated enzymatically using a protocol described previously (71). Isolated myocytes were investigated in a continuously superfused $(1.5 \mathrm{ml} / \mathrm{min}$, room temperature) recording chamber (vol, $1 \mathrm{ml}$ ) fixed to an inverted microscope. For TRPC6 current recording, we used the whole-cell voltage clamp technique with pipette resistances of 2-3 $\mathrm{M} \Omega$ when filled with internal solution. The junctional potential was corrected by zeroing the potential before the pipette tip touched the cell membrane. After the cell membrane was broken by application of additional suction, cell capacitance and series resistance were electrically compensated. After access was gained in the whole-cell voltage-clamp configuration, myocytes were allowed to equilibrate for $5 \mathrm{~min}$ utes with the internal solution before data were collected. Cells were depolarized by triangular voltage ramps from -120 to $+120 \mathrm{mV}$ (pulse duration, $200 \mathrm{~ms}$ ) from holding potential of $-60 \mathrm{mV}$ at a frequency of $1 \mathrm{~Hz}(42,72)$. As TRPC6 is a nonselective cation channel, current amplitude was analyzed as peak inward $(+10 \mathrm{mV})$ and peak outward current density $(+110 \mathrm{mV})$. After the current reached steady state, $10 \mathrm{nM} \mathrm{ET}-1$ was added in the external solution. The changes of the current were analyzed when the response of TRPC6 current to ET-1 reached its maximum (about $10 \mathrm{~min}$ ).

Solutions. Krebs-Ringer solution for cell isolation contained $35 \mathrm{mmol} / \mathrm{l}$ $\mathrm{NaCl}, 4.75 \mathrm{mmol} / \mathrm{l} \mathrm{KCl}, 1.19 \mathrm{mmol} / 1 \mathrm{KH}_{2} \mathrm{PO}_{4}, 16 \mathrm{mmol} / 1 \mathrm{Na}_{2} \mathrm{HPO}_{4}, 134$ $\mathrm{mmol} / \mathrm{l}$ sucrose, $25 \mathrm{mmol} / 1 \mathrm{NaCO}_{3}, 10 \mathrm{mmol} / \mathrm{l}$ glucose, $10 \mathrm{mmol} / 1$ HEPES, $\mathrm{pH} 7.4$ with $\mathrm{NaOH}$. The "KB" solution for storage of cells contained 10 $\mathrm{mmol} / \mathrm{l}$ taurine, $70 \mathrm{mmol} / \mathrm{l}$ glutamic acid, $25 \mathrm{mmol} / \mathrm{l} \mathrm{KCl}, 10 \mathrm{mmol} / \mathrm{l}$ $\mathrm{KH}_{2} \mathrm{PO}_{4}, 22 \mathrm{mmol} / 1$ glucose, $0.5 \mathrm{mmol} / 1$ EGTA, pH adjusted to 7.2 with $\mathrm{KOH}$. External solution for TRPC6 current recording contained $140 \mathrm{mM}$
$\mathrm{NaCl} ; 5 \mathrm{mM} \mathrm{CsCl} ; 2 \mathrm{mM} \mathrm{CaCl} 2 ; 1 \mathrm{mM} \mathrm{MgCl} 2 ; 10 \mathrm{mM}$ glucose; and $10 \mathrm{mM}$ HEPES, titrated to $\mathrm{pH} 7.4$ with $\mathrm{NaOH}$. Internal solution for TRPC6 current recording contained $120 \mathrm{mM} \mathrm{CsCl} ; 9.4 \mathrm{mM} \mathrm{NaCl} ; 1 \mathrm{mM} \mathrm{MgCl} ; 0.2$ $\mathrm{mM} \mathrm{Na}_{3} \mathrm{GTP}$, buffered at $100 \mathrm{nM}$ free $\mathrm{Ca}^{2+}$ with $10 \mathrm{mM}$ BAPTA; and 10 mM HEPES, $\mathrm{pH} 7.2$ with $\mathrm{CsOH}$.

Statistics. Data are presented as means \pm SEM. Unpaired Student's $t$ test was used for comparison between 2 groups, and ANOVA with post hoc Fisher's test was used for comparison among groups. Values of $P<0.05$ were considered significant.

Note added in proof. After submission of this manuscript, 2 publications appeared that provide further support for the involvement of TRPC channels in calcineurin-dependent cardiac hypertrophy $(72,73)$.

\section{Acknowledgments}

We thank Yongli Kong, Jun Cheng, and Guojin Huang for exceptional technical assistance. We thank Shmuel Muallen for scientific advice and gratefully acknowledge Beverly A. Rothermel and Eisaku Satoh for reagents. We also thank Alisha Tizenor for assistance with graphics. This work is supported by grants from the NIH, the Donald W. Reynolds Cardiovascular Clinical Research Center, the Muscular Dystrophy Association, the Robert A. Welch Foundation, and the Texas Advanced Technology Program to E.N. Olson and by a grant from the NIH to J.A. Hill. K. Kuwahara was supported by a research fellowship from the Uehara Memorial Foundation.

Received for publication December 19, 2005, and accepted in revised form September 19, 2006.

Address correspondence to: Eric N. Olson, University of Texas Southwestern Medical Center, 5323 Harry Hines Boulevard, Dallas, Texas 75390-9148, USA. Phone: (214) 648-1187; Fax: (214) 648-1196; E-mail: Eric.Olson@utsouthwestern.edu.
1. Olson, E.N., and Schneider, M.D. 2003. Sizing up the heart: development redux in disease. Genes Dev. 17:1937-1956.

2. Dorn, G.W., 2nd, and Force, T. 2005. Protein kinase cascades in the regulation of cardiac hypertrophy. J. Clin. Invest. 115:527-537. doi:10.1172/ JCI200524178.

3. Ahmad, F., Seidman, J.G., and Seidman, C.E. 2005. The genetic basis for cardiac remodeling. Annu. Rev. Genomics Hum. Genet. 6:185-216.

4. Nakao, K., Minobe, W., Roden, R., Bristow, M.R., and Leinwand, L.A. 1997. Myosin heavy chain gene expression in human heart failure. J. Clin. Invest. 100:2362-2370.

5. Wehrens, X.H., Lehnart, S.E., and Marks, A.R. 2005. Intracellular calcium release and cardiac disease. Annu. Rev. Physiol. 67:69-98.

6. Frey, N., and Olson, E.N. 2003. Cardiac hypertrophy: the good, the bad, and the ugly. Annu. Rev. Physiol. 65:45-79.

7. Houser, S.R., Piacentino, V., 3rd, and Weisser, J. 2000. Abnormalities of calcium cycling in the hypertrophied and failing heart. J. Mol. Cell. Cardiol. 32:1595-1607.

8. Hofmann, T., Schaefer, M., Schultz, G., and Gudermann, T. 2000. Transient receptor potential channels as molecular substrates of receptor-mediated cation entry. J. Mol. Med. 78:14-25.

9. Montell, C. 2005. The TRP superfamily of cation channels. Sci. STKE. 2005:re3.

10. Hunton, D.L., et al. 2002. Capacitative calcium entry contributes to nuclear factor of activated T-cells nuclear translocation and hypertrophy in cardiomyocytes. J. Biol. Chem. 277:14266-14273.

11. Uehara, A., Yasukochi, M., Imanaga, I., Nishi, M., and Takeshima, H. 2002. Store-operated Ca2+ entry uncoupled with ryanodine receptor and junctional membrane complex in heart muscle cells. Cell Calcium. 31:89-96.

12. Molkentin, J.D., et al. 1998. A calcineurin-dependent transcriptional pathway for cardiac hypertrophy. Cell. 93:215-228.

13. Crabtree, G.R., and Olson, E.N. 2002. NFAT signaling: choreographing the social lives of cells. Cell. 109(Suppl.):S67-S79.

14. Bueno, O.F., et al. 2002. Impaired cardiac hypertrophic response in calcineurin Abeta-deficient mice. Proc. Natl. Acad. Sci. U. S. A. 99:4586-4591.

15. De Windt, L.J., et al. 2001. Targeted inhibition of calcineurin attenuates cardiac hypertrophy in vivo. Proc. Natl. Acad. Sci. U. S. A. 98:3322-3327.

16. Rothermel, B., et al. 2000. A protein encoded within the Down syndrome critical region is enriched in striated muscles and inhibits calcineurin signaling. J. Biol. Chem. 275:8719-8725.

17. Wilkins, B.J., et al. 2002. Targeted disruption of NFATc3, but not NFATc4, reveals an intrinsic defect in calcineurin-mediated cardiac hypertrophic growth. Mol. Cell. Biol. 22:7603-7613.

18. Hill, J.A., et al. 2000. Cardiac hypertrophy is not a required compensatory response to short-term pressure overload. Circulation. 101:2863-2869.
19. Hill, J.A., et al. 2002. Targeted inhibition of calcineurin in pressure-overload cardiac hypertrophy. Preservation of systolic function. J. Biol. Chem. 277:10251-10255.

20. Feske, S., Draeger, R., Peter, H.H., Eichmann, K., and Rao, A. 2000. The duration of nuclear residence of NFAT determines the pattern of cytokine expression in human SCID T cells. J. Immunol. 165:297-305

21. Feske, S., Giltnane, J., Dolmetsch, R., Staudt, L.M., and Rao, A. 2001. Gene regulation mediated by calcium signals in T lymphocytes. Nat. Immunol. 2:316-324.

22. Minke, B., and Cook, B. 2002. TRP channel proteins and signal transduction. Physiol. Rev. 82:429-472.

23. Hofmann, T., Schaefer, M., Schultz, G., and Gudermann, T. 2002. Subunit composition of mammalian transient receptor potential channels in living cells. Proc. Natl. Acad. Sci. U. S. A. 99:7461-7466.

24. Strubing, C., Krapivinsky, G., Krapivinsky, L., and Clapham, D.E. 2003. Formation of novel TRPC channels by complex subunit interactions in embryonic brain. J. Biol. Chem. 278:39014-39019.

25. Hofmann, T., et al. 1999. Direct activation of human TRPC6 and TRPC3 channels by diacylglycerol. Nature. 397:259-263.

26. Rosenberg, P., et al. 2004. TRPC3 channels confer cellular memory of recent neuromuscular activity. Proc. Natl. Acad. Sci. U. S. A. 101:9387-9392.

27. Garcia, R.L., and Schilling, W.P. 1997. Differential expression of mammalian TRP homologues across 
tissues and cell lines. Biochem. Biophys. Res. Commun. 239:279-283.

28. Riccio, A., et al. 2002. mRNA distribution analysis of human TRPC family in CNS and peripheral tissues. Brain Res. Mol. Brain Res. 109:95-104.

29. Yang, J., et al. 2000. Independent signals control expression of the calcineurin inhibitory proteins MCIP1 and MCIP2 in striated muscles. Circ. Res. 87:E61-E68.

30. Karasseva, N., et al. 2003. Transcription enhancer factor 1 binds multiple muscle MEF2 and A/T-rich elements during fast-to-slow skeletal muscle fiber type transitions. Mol. Cell. Biol. 23:5143-5164.

31. McLean, B.G., Lee, K.S., Simpson, P.C., and Farrance, I.K. 2003. Basal and alpha1-adrenergic-induced activity of minimal rat betaMHC promoters in cardiac myocytes requires multiple TEF- 1 but not NFAT binding sites. J. Mol. Cell. Cardiol. 35:461-471.

32. Morimoto, T., et al. 1999. GATA-5 is involved in leukemia inhibitory factor-responsive transcription of the beta-myosin heavy chain gene in cardiac myocytes. J. Biol. Chem. 274:12811-12818.

33. Rothermel, B.A., et al. 2001. Myocyte-enriched calcineurin-interacting protein, MCIP1, inhibits cardiac hypertrophy in vivo. Proc. Natl. Acad. Sci. U. S. A. 98:3328-3333.

34. Wilkins, B.J., and Molkentin, J.D. 2004. Calciumcalcineurin signaling in the regulation of cardiac hypertrophy. Biochem. Biophys. Res. Commun. 322:1178-1191.

35. Inoue, R., et al. 2001. The transient receptor potential protein homologue TRP6 is the essential component of vascular alpha(1)-adrenoceptor-activated $\mathrm{Ca}(2+)$-permeable cation channel. Circ. Res. 88:325-332.

36. Jung, S., Strotmann, R., Schultz, G., and Plant, T.D. 2002. TRPC6 is a candidate channel involved in receptor-stimulated cation currents in A7r5 smooth muscle cells. Am. J. Physiol. Cell Physiol. 282:C347-C359.

37. Soboloff, J., et al. 2005. Role of endogenous TRPC6 channels in $\mathrm{Ca} 2+$ signal generation in $\mathrm{A} 7 \mathrm{r} 5$ smooth muscle cells. J. Biol. Chem. 280:39786-39794.

38. Venkatesh, N., et al. 2004. Chemical genetics to identify NFAT inhibitors: potential of targeting calcium mobilization in immunosuppression. Proc. Natl. Acad. Sci. U. S. A. 101:8969-8974.

39. Wilkins, B.J., et al. 2004. Calcineurin/NFAT coupling participates in pathological, but not physiological, cardiac hypertrophy. Circ. Res. 94:110-118.

40. Kunichika, N., et al. 2004. Bosentan inhibits transient receptor potential channel expression in pulmonary vascular myocytes. Am. J. Respir. Crit. Care Med. 170:1101-1107.

41. Yu, Y., et al. 2003. PDGF stimulates pulmonary vascular smooth muscle cell proliferation by upregulating TRPC6 expression. Am. J. Physiol. Cell Physiol. 284:C316-C330.
42. Dietrich, A., et al. 2005. Increased vascular smooth muscle contractility in TRPC6-/- mice. Mol. Cell. Biol. 25:6980-6989.

43. Mercer, J.C., et al. 2006. Large store-operated calcium selective currents due to co-expression of Orai1 or Orai2 with the intracellular calcium sensor, Stim1. J. Biol. Chem. 281:24979-24990.

44. Peinelt, C., et al. 2006. Amplification of CRAC current by STIM1 and CRACM1 (Orai1). Nat. Cell Biol. 8:771-773.

45. Prakriya, M., et al. 2006. Orai1 is an essential pore subunit of the CRAC channel. Nature 443:230-233.

46. Soboloff, J., et al. 2006. Orai1 and STIM reconstitute store-operated calcium channel function. J. Biol. Chem. 281:20661-20665.

47. Yeromin, A.V., et al. 2006. Molecular identification of the CRAC channel by altered ion selectivity in a mutant of Orai. Nature. 443:226-229.

48. Feske, S., et al. 2006. A mutation in Orai1 causes immune deficiency by abrogating CRAC channel function. Nature. 441:179-185.

49. Huang, G.N., et al. 2006. STIM1 carboxyl-terminus activates native SOC, I(crac) and TRPC1 channels. Nat. Cell Biol. 8:1003-1010.

50. Abraham, W.T., et al. 2002. Coordinate changes in myosin heavy chain isoform gene expression are selectively associated with alterations in dilated cardiomyopathy phenotype. Mol. Med. 8:750-760.

51. Miyata, S., Minobe, W., Bristow, M.R., and Leinwand, L.A. 2000. Myosin heavy chain isoform expression in the failing and nonfailing human heart. Circ. Res. 86:386-390.

52. Yasumura, Y., Takemura, K., Sakamoto, A., Kitakaze, M., and Miyatake, K. 2003. Changes in myocardial gene expression associated with beta-blocker therapy in patients with chronic heart failure. J. Card. Fail. 9:469-474.

53. Morkin, E. 2000. Control of cardiac myosin heavy chain gene expression. Microsc. Res. Tech. 50:522-531.

54. Delling, U., et al. 2000. A calcineurin-NFATc3dependent pathway regulates skeletal muscle differentiation and slow myosin heavy-chain expression. Mol. Cell. Biol. 20:6600-6611.

55. Oh, M., et al. 2005. Calcineurin is necessary for the maintenance but not embryonic development of slow muscle fibers. Mol. Cell. Biol. 25:6629-6638.

56. Serrano, A.L., et al. 2001. Calcineurin controls nerve activity-dependent specification of slow skeletal muscle fibers but not muscle growth. Proc. Natl. Acad. Sci. U. S. A. 98:13108-13113.

57. Tsika, R.W., McCarthy, J., Karasseva, N., Ou, Y., and Tsika, G.L. 2002. Divergence in species and regulatory role of beta -myosin heavy chain proximal promoter muscle-CAT elements. Am. J. Physiol. Cell Physiol. 283:C1761-C1775.

58. Wu, X., et al. 2006. Local InsP3-dependent perinuclear $\mathrm{Ca} 2+$ signaling in cardiac myocyte excitation transcription coupling. J. Clin. Invest. 116:675-682. doi:10.1172/JCI27374.

59. Schoenfeld, J.R., et al. 1998. Distinct molecular phenotypes in murine cardiac muscle development, growth, and hypertrophy. J. Mol. Cell. Cardiol. 30:2269-2280.

60. Sussman, M.A., et al. 2000. Altered focal adhesion regulation correlates with cardiomyopathy in mice expressing constitutively active rac1.J. Clin. Invest. 105:875-886.

61. Sussman, M.A., et al. 1998. Myofibril degeneration caused by tropomodulin overexpression leads to dilated cardiomyopathy in juvenile mice. J. Clin. Invest. 101:51-61.

62. Antos, C.L., et al. 2002. Activated glycogen synthase- 3 beta suppresses cardiac hypertrophy in vivo. Proc. Natl. Acad. Sci. U. S. A. 99:907-912.

63. Van Rooij, E., et al. 2004. MCIP1 overexpression suppresses left ventricular remodeling and sustains cardiac function after myocardial infarction. Circ. Res. 94:e18-e26.

64. Krenz, M., and Robbins, J. 2004. Impact of betamyosin heavy chain expression on cardiac function during stress. J. Am. Coll. Cardiol. 44:2390-2397.

65. Tardiff, J.C., et al. 2000. Expression of the beta (slow)-isoform of MHC in the adult mouse heart causes dominant-negative functional effects. Am. J. Physiol. Heart Circ. Physiol. 278:H412-H419.

66. Elliott, P., and McKenna, W.J. 2004. Hypertrophic cardiomyopathy. Lancet. 363:1881-1891.

67. Kamisago, M., et al. 2000. Mutations in sarcomere protein genes as a cause of dilated cardiomyopathy. N. Engl. J. Med. 343:1688-1696.

68. James, J., et al. 2005. Forced expression of alphamyosin heavy chain in the rabbit ventricle results in cardioprotection under cardiomyopathic conditions. Circulation. 111:2339-2346.

69. Frey, N., et al. 2004. Mice lacking calsarcin-1 are sensitized to calcineurin signaling and show accelerated cardiomyopathy in response to pathological biomechanical stress. Nat. Med. 10:1336-1343.

70. Wang, Y., Cheng, J., Joyner, R.W., Wagner, M.B., and Hill, J.A. 2006. Remodeling of early-phase repolarization: a mechanism of abnormal impulse conduction in heart failure. Circulation. 113:1849-1856.

71. Estacion, M., Sinkins, W.G., Jones, S.W., Applegate, M.A., and Schilling, W.P. 2006. Human TRPC6 expressed in HEK 293 cells forms non-selective cation channels with limited $\mathrm{Ca} 2+$ permeability. J. Physiol. 572:359-377.

72. Nakayama, H., Wilkin, B.J., Bodi, I., and Molkentin, J.D. 2006. Calcineurin-dependent caardiomyopathy is activated by TRPC in the adult mouse heart. FASEB J. 20:1660-1670.

73. Bush, E.W., et al. 2006. TRPC channels promote cardiomyocyte hypertrophy through activation of calcineurin signaling. J. Biol. Chem. In press. 